Supporting Information for:

\title{
A Tandem Aza-Claisen Rearrangement And Ring Closing Metathesis Reaction For The Synthesis of Cyclic Allylic Trichloroacetamides
}

\author{
Michael D. Swift and Andrew Sutherland*
}

WestChem, Department of Chemistry, The Joseph Black Building, University of Glasgow, Glasgow, UK G12 8QQ.Email: andrews@chem.gla.ac.uk

\section{Table of Contents}

1. General Experimental

S2

2. General Procedures

S2-S3

3. Experimental Procedures and Spectroscopic Data for All Compounds

S3-S9

4. References

S9

4. ${ }^{1} \mathrm{H}$ NMR and ${ }^{13} \mathrm{C}$ NMR Spectra of All Compounds

S10-S33 


\section{General Experimental}

All reagents and starting materials were obtained from commercial sources and used as received. All dry solvents were purified using a PureSolv 500 MD solvent purification system. All reactions were performed under an atmosphere of argon unless otherwise mentioned. Flash column chromatography was carried out using Fisher matrix silica 60. Macherey-Nagel aluminium-backed plates pre-coated with silica gel $60\left(\mathrm{UV}_{254}\right)$ were used for thin layer chromatography and were visualised by staining with $\mathrm{KMnO}_{4} \cdot{ }^{1} \mathrm{H}$ NMR and ${ }^{13} \mathrm{C}$ NMR spectra were recorded on a Bruker DPX 400 spectrometer with chemical shift values in ppm relative to tetramethylsilane as the standard. Infrared spectra were recorded using Golden Gate apparatus on a JASCO FTIR 410 spectrometer and mass spectra were obtained using a JEOL JMS-700 spectrometer. Melting points were determined on a Reichert platform melting point apparatus. Optical rotations were determined as solutions irradiating with the sodium $\mathrm{D}$ line $(\lambda=589 \mathrm{~nm})$ using an Autopol V polarimeter. $[\alpha]_{\mathrm{D}}$ values are given in units $10^{-1} \mathrm{deg}^{2} \mathrm{gm}^{-1}$. The chiral GC and HPLC methods were calibrated with their corresponding racemic mixtures.

\section{General Procedure 1: One Pot Swern Oxidation-Horner/Wadsworth/Emmons Reaction.}

Dimethyl sulfoxide (2.5 equiv.) was added to a stirred solution of oxalyl chloride (1.4 equiv.) in dichloromethane $(100 \mathrm{~mL})$ at $-78^{\circ} \mathrm{C}$. The reaction mixture was stirred for $0.3 \mathrm{~h}$ before the alcohol (1.0 equiv.) in dichloromethane $(50 \mathrm{~mL})$ was slowly added. The mixture was stirred for a further $0.3 \mathrm{~h}$ before triethylamine ( 5 equiv.) was added. This reaction mixture was stirred for $0.5 \mathrm{~h}$ at -78 ${ }^{\circ} \mathrm{C}$ and then allowed to warm to room temperature and stirred for a further $2 \mathrm{~h}$. Meanwhile, a solution of lithium chloride (1.8 equiv.), triethyl phosphonoacetate (1.5 equiv.) and 1,8diazabicyclo[5,4,0]undec-7-ene (1.5 equiv.) in acetonitrile $(100 \mathrm{~mL})$ was prepared and stirred for $1.0 \mathrm{~h}$. The Swern solution was concentrated in vacuo, then the Horner/Wadsworth/Emmons solution was added and the reaction mixture was stirred at room temperature overnight. The reaction was quenched with a saturated solution of ammonium chloride $(50 \mathrm{~mL})$ and concentrated to give an orange residue, which was then extracted with diethyl ether ( 4 x $75 \mathrm{~mL}$ ). The organic layers were combined, dried $\left(\mathrm{MgSO}_{4}\right)$ and concentrated to give an orange oil. Purification by flash column chromatography using diethyl ether / petroleum ether as eluent gave the product.

General Procedure 2: DIBAL-H Reduction To Allylic Alcohol. The ester (1.0 equiv.) was dissolved in diethyl ether $(100 \mathrm{~mL})$ and cooled to $-78{ }^{\circ} \mathrm{C}$. DIBAL-H (1 M in hexane) (2.2 equiv.) was added dropwise and the reaction mixture was stirred at $-78^{\circ} \mathrm{C}$ for $3 \mathrm{~h}$, before warming to room 
temperature. The solution was cooled to $0{ }^{\circ} \mathrm{C}$ and quenched by the addition of a saturated solution of ammonium chloride $(10 \mathrm{~mL})$ and warmed to room temperature with vigorous stirring over $1 \mathrm{~h}$ producing a white precipitate. The precipitate was filtered through a pad of Celite ${ }^{\circledR}$ and washed with diethyl ether $(400 \mathrm{~mL})$. The filtrate was then dried $\left(\mathrm{MgSO}_{4}\right)$ and concentrated in vacuo. Purification was carried out by flash column chromatography eluting with diethyl ether / petroleum ether.

\section{General Procedure 3: Synthesis Of Allylic Trichloroacetimidate And Subsequent Tandem} Aza-Claisen Rearrangement / Ring Closing Metathesis. Allylic alcohol (1 equiv.) was dissolved in dichloromethane $(20 \mathrm{~mL})$ and cooled to $0{ }^{\circ} \mathrm{C}$. 1,8-Diazabicyclo[5.4.0]undec-7-ene (0.25 equiv.) was added to the solution followed by trichloroacetonitrile (1.5 equiv.). The solution was then warmed to room temperature and stirred for $2 \mathrm{~h}$. The reaction mixture was filtered through a short pad of silica gel and washed with diethyl ether $(100 \mathrm{~mL})$. The resulting filtrate was then concentrated to give the allylic trichloroacetimidate, which was used without further purification. Allylic trichloroacetimidate (1 equiv.) was then dissolved in dichloromethane $(10 \mathrm{~mL})$ under an argon atmosphere. The rearrangement catalyst ( 0.1 equiv., $10 \mathrm{~mol} \%$ ) was added to the solution and the reaction mixture was stirred at room temperature for $3 \mathrm{~h}$. Grubb's catalyst ( $1^{\text {st }}$ Generation) $(0.1$ equiv., $10 \mathrm{~mol} \%$ ) was then added and the reaction mixture was heated under reflux overnight. The mixture was cooled to room temperature and then filtered through a short pad of Celite ${ }^{\circledR}$ and washed with diethyl ether $(100 \mathrm{~mL})$. Concentration of the filtrate followed by flash column chromatography gave the cyclic allylic amides as white solids.

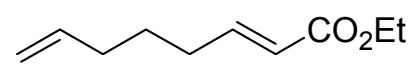

Ethyl (2E)-2,7-octadienoate 2. ${ }^{1}$ Reaction was carried out according to general procedure 1, using 5-hexen-1-ol 1 (4.0 g, $0.04 \mathrm{~mol}$ ). Flash column chromatography (petroleum ether/ diethyl ether 10:1) yielded ethyl (2E)-2,7-octadienoate 2 (4.6 g, 69\% yield) as a yellow oil. Spectroscopic data entirely consistent with literature. ${ }^{1} \mathrm{H}$ NMR $\left(400 \mathrm{MHz}, \mathrm{CDCl}_{3}\right) \delta 1.28(\mathrm{t}, J=7.1 \mathrm{~Hz}, 3 \mathrm{H}), 1.56$ (quin, $J=7.2 \mathrm{~Hz}, 2 \mathrm{H}), 2.06-2.12(\mathrm{~m}, 2 \mathrm{H}), 2.18-2.25$ (m, 2H), 4.19 (q, $J=7.1 \mathrm{~Hz}, 2 \mathrm{H}), 4.96-5.06$ (m, $2 \mathrm{H}), 5.73-5.86(\mathrm{~m}, 2 \mathrm{H}), 6.95(\mathrm{dt}, J=15.5,6.9 \mathrm{~Hz}, 1 \mathrm{H}) ;{ }^{13} \mathrm{C} \mathrm{NMR}\left(100 \mathrm{MHz}, \mathrm{CDCl}_{3}\right) \delta 14.3\left(\mathrm{CH}_{3}\right)$, $27.1\left(\mathrm{CH}_{2}\right), 31.5\left(\mathrm{CH}_{2}\right), 33.1\left(\mathrm{CH}_{2}\right), 60.2\left(\mathrm{CH}_{2}\right), 115.1\left(\mathrm{CH}_{2}\right), 121.5(\mathrm{CH}), 138.0(\mathrm{CH}), 149.0(\mathrm{CH})$, 166.7 (C); HRMS (CI) $m / z$ 169.1232 (calcd for $\mathrm{C}_{10} \mathrm{H}_{17} \mathrm{O}_{2}: 169.1229, \mathrm{MH}^{+}$). 


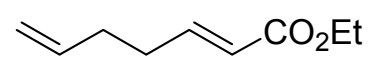

Ethyl (2E)-2,6-heptadienoate. ${ }^{2}$ Reaction was carried out according to general procedure 1, using 4penten-1-ol (0.5 g, $5.8 \mathrm{mmol}$ ). Flash column chromatography (petroleum ether/ diethyl ether 20:1) yielded ethyl (2E)-2,6-heptadienoate $(0.69 \mathrm{~g}, 77 \%$ yield) as a yellow oil. Spectroscopic data consistent with literature. ${ }^{2}{ }^{\mathrm{H}} \mathrm{NMR}\left(400 \mathrm{MHz}, \mathrm{CDCl}_{3}\right) \delta 1.29(\mathrm{t}, J=7.1 \mathrm{~Hz}, 3 \mathrm{H}), 1.99-2.25(\mathrm{~m}, 2 \mathrm{H})$, 2.27-2.34 (m, 2H), 4.19 (q, $J=7.1 \mathrm{~Hz}, 2 \mathrm{H}), 5.01(\mathrm{dq}, J=10.5,1.5 \mathrm{~Hz}, 1 \mathrm{H}), 5.06(\mathrm{dq}, J=17.0,1.5$ $\mathrm{Hz}, 1 \mathrm{H}), 5.75-5.86(\mathrm{~m}, 2 \mathrm{H}), 6.96(\mathrm{dt}, J=15.6,6.8 \mathrm{~Hz}, 1 \mathrm{H}) ;{ }^{13} \mathrm{C} \mathrm{NMR}\left(100 \mathrm{MHz}, \mathrm{CDCl}_{3}\right) \delta 14.3$ $\left(\mathrm{CH}_{3}\right), 31.5\left(\mathrm{CH}_{2}\right), 32.0\left(\mathrm{CH}_{2}\right), 60.2\left(\mathrm{CH}_{2}\right), 115.5\left(\mathrm{CH}_{2}\right), 121.7(\mathrm{CH}), 137.1(\mathrm{CH}), 148.3(\mathrm{CH})$, 166.7 (C); HRMS (CI) m/z 155.1075 (calcd for $\mathrm{C}_{9} \mathrm{H}_{15} \mathrm{O}_{2}: 155.1072, \mathrm{MH}^{+}$).

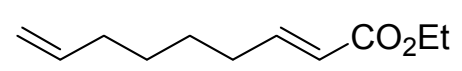

Ethyl (2E)-2,8-nonadienoate. ${ }^{3}$ Reaction was carried out according to general procedure 1, using 6hepten-1-ol (1.0 g, $8.8 \mathrm{mmol})$. Flash column chromatography (petroleum ether/ diethyl ether 97:3) yielded ethyl (2E)-2,8-nonadienoate (1.15 g, 72\% yield) as a yellow oil. Spectroscopic data consistent with literature. ${ }^{3}{ }^{1} \mathrm{H}$ NMR (400 MHz, $\left.\mathrm{CDCl}_{3}\right) \delta 1.29(\mathrm{t}, J=7.1 \mathrm{~Hz}, 3 \mathrm{H}), 1.38-1.52(\mathrm{~m}, 4 \mathrm{H})$, 2.06 (qt, $J=7.2,1.6 \mathrm{~Hz}, 2 \mathrm{H}), 2.21(\mathrm{qd}, J=7.0,1.5 \mathrm{~Hz}, 2 \mathrm{H}), 4.18$ (q, $J=7.1 \mathrm{~Hz}, 2 \mathrm{H}), 4.95(\mathrm{~m}, 1 \mathrm{H})$, $5.00(\mathrm{dq}, J=17.1,1.6 \mathrm{~Hz}, 1 \mathrm{H}), 5.74-5.85(\mathrm{~m}, 2 \mathrm{H}), 6.96(\mathrm{dt}, J=15.6,7.0 \mathrm{~Hz}, 1 \mathrm{H}) ;{ }^{13} \mathrm{C} \mathrm{NMR}(100$ $\left.\mathrm{MHz}, \mathrm{CDCl}_{3}\right) \delta 14.3\left(\mathrm{CH}_{3}\right), 27.4\left(\mathrm{CH}_{2}\right), 28.3\left(\mathrm{CH}_{2}\right), 32.0\left(\mathrm{CH}_{2}\right), 33.5\left(\mathrm{CH}_{2}\right), 60.2\left(\mathrm{CH}_{2}\right), 114.7$ $\left(\mathrm{CH}_{2}\right), 121.3(\mathrm{CH}), 138.6(\mathrm{CH}), 149.3(\mathrm{CH}), 166.8(\mathrm{C})$; HRMS (CI) $\mathrm{m} / z 183.1382$ (calcd for $\left.\mathrm{C}_{11} \mathrm{H}_{19} \mathrm{O}_{2}: 183.1385, \mathrm{MH}^{+}\right)$.

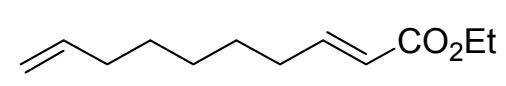

Ethyl (2E)-2,9-decadienoate. ${ }^{4}$ Reaction was carried out according to general procedure 1, using 7octen-1-ol (1.5 g, $11.7 \mathrm{mmol}$ ). Flash column chromatography (petroleum ether/ diethyl ether 50:1) yielded ethyl (2E)-2,9-decadienoate (2.06 g, 90\% yield) as a pale yellow oil. Spectroscopic data consistent with literature. ${ }^{4}{ }^{1} \mathrm{H}$ NMR (400 MHz, $\left.\mathrm{CDCl}_{3}\right) \delta 1.29(\mathrm{t}, J=7.1 \mathrm{~Hz}, 3 \mathrm{H}), 1.31-1.51(\mathrm{~m}, 6 \mathrm{H})$, 2.05 (qt, $J=7.1,1.3 \mathrm{~Hz}, 2 \mathrm{H}), 2.21(\mathrm{qd}, J=7.0,1.5 \mathrm{~Hz}, 2 \mathrm{H}), 4.18$ (q, $J=7.1 \mathrm{~Hz}, 2 \mathrm{H}), 4.94(\mathrm{~m}, 1 \mathrm{H})$, $4.99(\mathrm{dq}, J=17.1,1.3 \mathrm{~Hz}, 1 \mathrm{H}), 5.75-5.86(\mathrm{~m}, 2 \mathrm{H}), 6.96(\mathrm{dt}, J=15.6,7.0 \mathrm{~Hz}, 1 \mathrm{H}) ;{ }^{13} \mathrm{C}$ NMR $(100$ $\left.\mathrm{MHz}, \mathrm{CDCl}_{3}\right) \delta 14.3\left(\mathrm{CH}_{3}\right), 27.9\left(\mathrm{CH}_{2}\right), 28.6\left(\mathrm{CH}_{2}\right), 28.7\left(\mathrm{CH}_{2}\right)$ 32.2 $\left(\mathrm{CH}_{2}\right), 33.7\left(\mathrm{CH}_{2}\right), 60.2\left(\mathrm{CH}_{2}\right)$, $114.4\left(\mathrm{CH}_{2}\right), 121.3(\mathrm{CH}), 138.9(\mathrm{CH}), 149.4(\mathrm{CH}), 166.8(\mathrm{C})$; HRMS (CI) $m / z 197.1540$ (calcd for $\left.\mathrm{C}_{12} \mathrm{H}_{21} \mathrm{O}_{2}: 197.1542, \mathrm{MH}^{+}\right)$. 


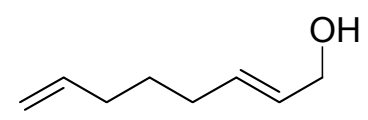

(2E)-Octa-2,7-dien-1-ol 3. ${ }^{1}$ The reaction was carried out according to general procedure 2, using ethyl (2E)-2,7-octadienoate 2 (1.0 g, $6.0 \mathrm{mmol})$. Flash column chromatography (petroleum ether/ diethyl ether 1:4) yielded (2E)-octa-2,7-dien-1-ol 3 (0.65 g, 87\% yield) as a colourless oil. Spectroscopic data consistent with literature. ${ }^{1}{ }^{1} \mathrm{H}$ NMR $\left(400 \mathrm{MHz}, \mathrm{CDCl}_{3}\right) \delta 1.34$ (br s, 1H), 1.48 (quin, $J=7.2 \mathrm{~Hz}, 2 \mathrm{H}), 2.03-2.10(\mathrm{~m}, 4 \mathrm{H}), 4.09$ (d, $J=4.6 \mathrm{~Hz}, 2 \mathrm{H}), 4.94-4.97$ (m, 1H), 5.01 (dq, $J=$ 17.0, 1.7 Hz, 1H), 5.60-5.73 (m, 2H), 5.80 (ddt, $J=17.0,10.2,6.7 \mathrm{~Hz}, 1 \mathrm{H}) ;{ }^{13} \mathrm{C} \mathrm{NMR}(100 \mathrm{MHz}$, $\left.\mathrm{CDCl}_{3}\right) \delta 28.3\left(\mathrm{CH}_{2}\right), 31.6\left(\mathrm{CH}_{2}\right), 33.2\left(\mathrm{CH}_{2}\right), 63.8\left(\mathrm{CH}_{2}\right), 114.6\left(\mathrm{CH}_{2}\right), 129.2(\mathrm{CH}), 133.0(\mathrm{CH})$, $138.6(\mathrm{CH}) ; \mathrm{MS}(\mathrm{CI}) m / z 127\left(\mathrm{MH}^{+}, 5 \%\right), 109$ (100), 95 (15), 67 (47).

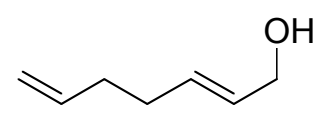

(2E)-Hepta-2,6-dien-1-ol. ${ }^{2}$ The reaction was carried out according to general procedure 2, using ethyl (2E)-2,6-heptadienoate $(1.54 \mathrm{~g}, 10.0 \mathrm{mmol})$. Flash column chromatography (petroleum ether/ diethyl ether 7:3) yielded (2E)-hepta-2,6-dien-1-ol $(0.92 \mathrm{~g}, 82 \%$ yield) as a colourless oil. Spectroscopic data consistent with literature. ${ }^{2} \mathrm{H}_{\mathrm{NMR}}\left(400 \mathrm{MHz}, \mathrm{CDCl}_{3}\right) \delta 1.42$ (br s, 1H), 2.10$2.15(\mathrm{~m}, 4 \mathrm{H}), 4.08$ (d, $J=4.8 \mathrm{~Hz}, 2 \mathrm{H}), 4.96-4.99(\mathrm{~m}, 1 \mathrm{H}), 5.00-5.06(\mathrm{~m}, 1 \mathrm{H}), 5.64-5.71(\mathrm{~m}, 2 \mathrm{H})$, 5.76-5.87 (m, 1H); ${ }^{13} \mathrm{C}$ NMR (100 MHz, $\left.\mathrm{CDCl}_{3}\right) \delta 31.5\left(\mathrm{CH}_{2}\right), 33.3\left(\mathrm{CH}_{2}\right), 63.7\left(\mathrm{CH}_{2}\right), 114.9\left(\mathrm{CH}_{2}\right)$, $129.4(\mathrm{CH}), 132.4(\mathrm{CH}), 138.1(\mathrm{CH})$; HRMS (CI) $\mathrm{m} / z 113.0964$ (calcd for $\mathrm{C}_{7} \mathrm{H}_{13} \mathrm{O}: 113.0966$, $\left.\mathrm{MH}^{+}\right)$.

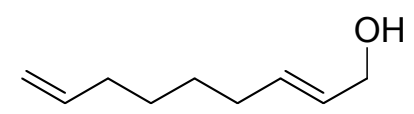

(2E)-Nona-2,8-dien-1-ol. ${ }^{3}$ The reaction was carried out according to general procedure 2, using ethyl (2E)-2,8-nonadienoate (1.0 g, $5.5 \mathrm{mmol})$. Flash column chromatography (petroleum ether/ diethyl ether 7:3) yielded (2E)-nona-2,8-dien-1-ol $(0.77 \mathrm{~g}, 100 \%$ yield) as a colourless oil. Spectroscopic data consistent with literature. ${ }^{3} \mathrm{H}$ NMR (400 MHz, $\left.\mathrm{CDCl}_{3}\right) \delta 1.22$ (br s, 1H), 1.38$1.42(\mathrm{~m}, 4 \mathrm{H}), 2.02-2.10(\mathrm{~m}, 4 \mathrm{H}), 4.09$ (t, $J=4.9 \mathrm{~Hz}, 2 \mathrm{H}), 4.92-4.96$ (m, 1H), 5.00 (dq, $J=17.0,1.6$ $\mathrm{Hz}, 1 \mathrm{H}), 5.60-5.74(\mathrm{~m}, 2 \mathrm{H}), 5.81$ (ddt, $J=17.0,10.2,6.7 \mathrm{~Hz}, 1 \mathrm{H}) ;{ }^{13} \mathrm{C} \mathrm{NMR}\left(100 \mathrm{MHz}, \mathrm{CDCl}_{3}\right) \delta$ $26.9\left(\mathrm{CH}_{2}\right), 27.1\left(\mathrm{CH}_{2}\right), 30.6\left(\mathrm{CH}_{2}\right), 32.2\left(\mathrm{CH}_{2}\right), 62.4\left(\mathrm{CH}_{2}\right), 112.9\left(\mathrm{CH}_{2}\right), 127.5(\mathrm{CH}), 131.9(\mathrm{CH})$, $137.4(\mathrm{CH})$; HRMS (CI) $m / z 123.1169$ (calcd for $\mathrm{C}_{9} \mathrm{H}_{15}: 123.1174, \mathrm{MH}^{+}-\mathrm{H}_{2} \mathrm{O}$ ). 


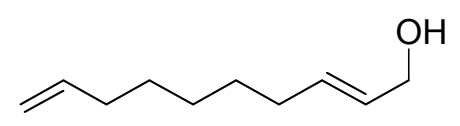

(2E)-Deca-2,9-dien-1-ol. ${ }^{5}$ The reaction was carried out according to general procedure 2, using ethyl (2E)-2,9-decadienoate $(1.5 \mathrm{~g}, 7.7 \mathrm{mmol})$. Flash column chromatography (petroleum ether/ diethyl ether 7:3) yielded (2E)-deca-2,9-dien-1-ol $(0.97 \mathrm{~g}, 81 \%$ yield) as a colourless oil. Spectroscopic data consistent with literature. ${ }^{5} \mathrm{H}$ NMR $\left(400 \mathrm{MHz}, \mathrm{CDCl}_{3}\right) \delta$ 1.27-1.45 (m, 7H), 2.01-2.09 (m, 4H), 4.09 (d, $J=4.6 \mathrm{~Hz}, 2 \mathrm{H}), 4.92-4.96$ (m, 1H), 4.97-5.04 (m, 1H), 5.58-5.74 (m, $2 \mathrm{H}), 5.81$ (ddt, $J=17.1,10.1,6.7 \mathrm{~Hz}, 1 \mathrm{H}) ;{ }^{13} \mathrm{C} \mathrm{NMR}\left(100 \mathrm{MHz}, \mathrm{CDCl}_{3}\right) \delta 28.7\left(\mathrm{CH}_{2}\right), 28.8\left(\mathrm{CH}_{2}\right)$, $29.0\left(\mathrm{CH}_{2}\right), 32.2\left(\mathrm{CH}_{2}\right), 33.8\left(\mathrm{CH}_{2}\right), 63.9\left(\mathrm{CH}_{2}\right), 114.3\left(\mathrm{CH}_{2}\right), 128.9(\mathrm{CH}), 133.5(\mathrm{CH}), 139.1(\mathrm{CH})$; HRMS (CI) $m / z 137.1324$ (calcd for $\mathrm{C}_{10} \mathrm{H}_{17}: 137.1330, \mathrm{MH}^{+}-\mathrm{H}_{2} \mathrm{O}$ ).<smiles>O=C(NC1C=CCCC1)C(Cl)(Cl)Cl</smiles>

1-(2',2',2'-Trichloromethylcarbonylamino)cyclohexa-2-ene $6 .^{6}$ The reaction was carried out according general procedure 3 using (2E)-octa-2,7-dien-1-ol 3 (0.1 g, $0.8 \mathrm{mmol})$ and bis(acetonitrile)palladium(II) chloride $(0.02 \mathrm{~g}, 0.08 \mathrm{mmol})$ for the aza-Claisen rearrangement. Purification by flash column chromatography (elution with petroleum ether/ diethyl ether 97:3) gave 1-(2',2',2'-trichloromethylcarbonylamino)cyclohexa-2-ene 6 as a white solid $(0.17 \mathrm{~g}, 89 \%$ yield over 3 steps). mp 85-86 ${ }^{\circ} \mathrm{C}$, lit. $.{ }^{6} 85.5-87{ }^{\circ} \mathrm{C} ;{ }^{1} \mathrm{H} \mathrm{NMR}\left(400 \mathrm{MHz}, \mathrm{CDCl}_{3}\right) \delta 1.61-1.73(\mathrm{~m}, 3 \mathrm{H})$, 1.94-2.02 (m, 1H), 2.04-2.14 (m, 2H), 4.42-4.50 (m, 1H), 5.65 (ddt, $J=10.0,4.0,2.2 \mathrm{~Hz}, 1 \mathrm{H}), 5.98$ $(\mathrm{dtd}, J=10.0,4.0,1.9 \mathrm{~Hz}, 1 \mathrm{H}), 6.60($ br s, $1 \mathrm{H}) ;{ }^{13} \mathrm{C} \mathrm{NMR}\left(100 \mathrm{MHz}, \mathrm{CDCl}_{3}\right) \delta 19.4\left(\mathrm{CH}_{2}\right), 24.7$ $\left(\mathrm{CH}_{2}\right), 28.6\left(\mathrm{CH}_{2}\right), 46.9(\mathrm{CH}), 92.7(\mathrm{C}), 125.7(\mathrm{CH}), 132.7(\mathrm{CH}), 161.1(\mathrm{C})$; HRMS $(\mathrm{CI}) \mathrm{m} / z$ 261.0144 (calcd for $\mathrm{C}_{8} \mathrm{H}_{14} \mathrm{~N}_{2} \mathrm{O}^{35} \mathrm{Cl}_{2}{ }^{37} \mathrm{Cl}: 261.0143, \mathrm{M}+\mathrm{NH}_{4}{ }^{+}$).

\section{Synthesis of 1-(2',2',2'-Trichloromethylcarbonylamino)cyclohexa-2-ene $6{ }^{6}$, using Grubbs'} catalyst ( $2^{\text {nd }}$ Generation). The reaction was carried out according general procedure 3 using $(2 E)$ octa-2,7-dien-1-ol 3 (0.08 g, $0.6 \mathrm{mmol})$, bis(acetonitrile)palladium(II) chloride (0.015 g, 0.06 mmol) for the aza-Claisen rearrangement and Grubbs' catalyst ( $2^{\text {nd }}$ Generation $)(0.05 \mathrm{~g}, 0.06 \mathrm{mmol})$ for the RCM reaction. Purification by flash column chromatography (elution with petroleum ether/ diethyl ether 97:3) gave 1-(2',2',2'-trichloromethylcarbonylamino)cyclohexa-2-ene 6, as a white solid $(0.14 \mathrm{~g}, 95 \%$ yield over 3 steps). Spectroscopic data as described above. 
Synthesis of 1-(2',2',2'-Trichloromethylcarbonylamino)cyclohexa-2-ene $\quad 6,^{6} \quad$ using Grubbs'/Hoveyda catalyst $\left(2^{\text {nd }}\right.$ Generation). The reaction was carried out according general procedure 3 using (2E)-octa-2,7-dien-1-ol 3 (0.08 g, $0.6 \mathrm{mmol})$, bis(acetonitrile)palladium(II) chloride $(0.015 \mathrm{~g}, 0.06 \mathrm{mmol})$ for the aza-Claisen rearrangement and Grubbs'/Hoveyda catalyst $\left(2^{\text {nd }}\right.$ Generation) (0.04 g, $0.06 \mathrm{mmol})$ for the RCM reaction. Purification by flash column chromatography (elution with petroleum ether/ diethyl ether 97:3) gave 1-(2',2',2'trichloromethylcarbonylamino)cyclohexa-2-ene 6, as a white solid (0.14 g, 95\% yield over 3 steps). Spectroscopic data as described above.<smiles>O=C(NC1C=CCC1)C(Cl)(Cl)Cl</smiles>

1-(2',2',2'-Trichloromethylcarbonylamino)cyclopenta-2-ene 8. The reaction was carried out according general procedure 3 using (2E)-hepta-2,6-dien-1-ol (0.1 g, $0.9 \mathrm{mmol})$ and bis(acetonitrile)palladium(II) chloride $(0.04 \mathrm{~g}, 0.18 \mathrm{mmol})$ for the aza-Claisen rearrangement. Purification by flash column chromatography (elution with petroleum ether/ diethyl ether 24:1) gave 1-(2',2',2' trichloromethylcarbonylamino)cyclopenta-2-ene $\mathbf{8}$ as a white solid $(0.17 \mathrm{~g}, 84 \%$ yield over 3 steps). mp 80-81 ${ }^{\circ} \mathrm{C}$; IR ( NaCl) 3291, 1684, 1528, $823 \mathrm{~cm}^{-1}$; ${ }^{1} \mathrm{H}$ NMR (400 MHz, $\left.\mathrm{CDCl}_{3}\right) \delta$ 1.67-1.74 (m, 1H) 2.34-2.57 (m, 3H), 4.92-5.01 (m, 1H), 5.72-5.76 (m, 1H), 6.05-6.09 (m, 1H), $6.68($ br s, $1 \mathrm{H}) ;{ }^{13} \mathrm{C}$ NMR (100 MHz, $\left.\mathrm{CDCl}_{3}\right)$ \& $28.9\left(\mathrm{CH}_{2}\right), 29.4\left(\mathrm{CH}_{2}\right), 56.0(\mathrm{CH}), 90.8$ (C), $127.5(\mathrm{CH}), 134.6(\mathrm{CH}), 159.3(\mathrm{C})$; HRMS (CI) $\mathrm{m} / z 229.9718$ (calcd for $\mathrm{C}_{7} \mathrm{H}_{9} \mathrm{NO}^{35} \mathrm{Cl}_{2}{ }^{37} \mathrm{Cl}$ : 229.9721, $\left.\mathrm{MH}^{+}\right)$.<smiles>O=C(NC1C=CCCCC1)C(Cl)(Cl)Cl</smiles>

1-(2',2',2'-Trichloromethylcarbonylamino)cyclohepta-2-ene $10 .^{7}$ The reaction was carried out according general procedure 3 (at a concentration of $0.005 \mathrm{M})$ using (2E)-nona-2,8-dien-1-ol $(0.1 \mathrm{~g}$, $0.7 \mathrm{mmol})$ and bis(acetonitrile)palladium(II) chloride $(0.02 \mathrm{~g}, 0.08 \mathrm{mmol})$ for the aza-Claisen rearrangement. Purification by flash column chromatography (elution with petroleum ether/ dichloromethane 5:2) gave 1-(2',2',2'-trichloromethylcarbonylamino)cyclohepta-2-ene 10, as a white solid (0.17 g, 93\% yield over 3 steps). mp 104-105 ${ }^{\circ} \mathrm{C}$, lit. ${ }^{7} 105{ }^{\circ} \mathrm{C} ;{ }^{1} \mathrm{H} \mathrm{NMR}$ (400 MHz, 
$\left.\mathrm{CDCl}_{3}\right) \delta$ 1.39-1.49 (m, 1H), 1.66-1.79 (m, 3H), 1.85-1.98 (m, 2H), 2.10-2.29 (m, 2H), 4.54-4.62 (m, 1H), 5.55-5.62 (m, 1H), 5.88-5.95 (m, 1H), 6.72 (br s, 1H); ${ }^{13} \mathrm{C}$ NMR (100 MHz, $\left.\mathrm{CDCl}_{3}\right) \delta 24.6$ $\left(\mathrm{CH}_{2}\right), 25.2\left(\mathrm{CH}_{2}\right), 26.5\left(\mathrm{CH}_{2}\right), 31.1\left(\mathrm{CH}_{2}\right), 50.5(\mathrm{CH}), 90.8(\mathrm{C}), 130.5(\mathrm{CH}), 131.8(\mathrm{CH}), 158.8(\mathrm{C})$; HRMS (CI) $m / z 256.0060$ (calcd for $\mathrm{C}_{9} \mathrm{H}_{13} \mathrm{NO}^{35} \mathrm{Cl}_{3}: 256.0063, \mathrm{MH}^{+}$).

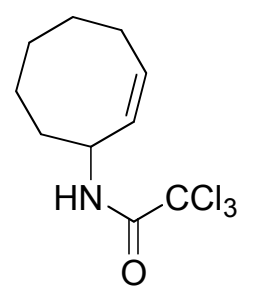

1-(2',2',2'-Trichloromethylcarbonylamino)cycloocta-2-ene 12. The reaction was carried out according general procedure 3 (at a concentration of $0.0013 \mathrm{M}$ ) using (2E)-deca-2,8-dien-1-ol (0.1 g, $0.7 \mathrm{mmol})$. Bis(acetonitrile)palladium(II) chloride $(0.008 \mathrm{~g}, 0.03 \mathrm{mmol})$ was used to catalyze the aza-Claisen rearrangement and Grubbs' catalyst ( ${ }^{\text {nd }}$ Generation) (0.05 g, $0.06 \mathrm{mmol}, 20 \mathrm{~mol} \%$ ) was used to catalyze the RCM. Purification by flash column chromatography (elution with petroleum ether/ dichloromethane 5:2) gave 1-(2',2',2'-trichloromethylcarbonylamino)cycloocta-2-ene 12, as a white solid (0.044 g, 62\% yield over 3 steps). mp 126-127 ${ }^{\circ} \mathrm{C}$; IR ( $\left.\mathrm{NaCl}\right) 3314,1684,1529,824$ $\mathrm{cm}^{-1}$; ${ }^{1} \mathrm{H}$ NMR (400 MHz, $\mathrm{CDCl}_{3}$ ) $\delta$ 1.31-1.78 (m, 7H), 1.98 (ddt, J= 13.1, 8.9, $\left.4.4 \mathrm{~Hz}, 1 \mathrm{H}\right), 2.11$ $2.20(\mathrm{~m}, 1 \mathrm{H}), 2.25-2.36(\mathrm{~m}, 1 \mathrm{H}), 4.79(\mathrm{~m}, 1 \mathrm{H}), 5.35(\mathrm{~m}, 1 \mathrm{H}), 5.79(\mathrm{dtd}, J=10.5,7.5,1.4 \mathrm{~Hz}, 1 \mathrm{H})$, 6.65 (br s, 1H); ${ }^{13} \mathrm{C}$ NMR (100 MHz, $\left.\mathrm{CDCl}_{3}\right)$ ठ $22.1\left(\mathrm{CH}_{2}\right), 23.9\left(\mathrm{CH}_{2}\right), 24.4\left(\mathrm{CH}_{2}\right), 26.8\left(\mathrm{CH}_{2}\right)$, $33.7\left(\mathrm{CH}_{2}\right), 47.9(\mathrm{CH}), 90.7(\mathrm{C}), 127.3(\mathrm{CH}), 129.5(\mathrm{CH}), 158.9(\mathrm{C})$; HRMS (CI) m/z 270.0216 (calcd for $\mathrm{C}_{10} \mathrm{H}_{15} \mathrm{NO}^{35} \mathrm{Cl}_{3}: 270.0219, \mathrm{MH}^{+}$).<smiles>O=C(NC1C=CCCC1)C(Cl)(Cl)Cl</smiles>

(1S)-(2',2',2'-Trichloromethylcarbonylamino)cyclohexa-2-ene 14. The reaction was carried out according general procedure 3 using (2E)-octa-2,7-dien-1-ol $3(0.08 \mathrm{~g}, 0.6 \mathrm{mmol})$ and $(S)$-COP-Cl $(0.04 \mathrm{~g}, 0.03 \mathrm{mmol})$ for the aza-Claisen rearrangement. Purification by flash column chromatography (elution with petroleum ether/ diethyl ether 97:3) gave (1S)-(2',2',2'trichloromethylcarbonylamino)cyclohexa-2-ene $\mathbf{1 4}$ as a white solid $(0.15 \mathrm{~g}, 90 \%$ yield over 3 steps). $88 \%$ ee determined by HPLC analysis using CHIRALPAK IB column $(0.5 \%$ PrOH/hexane at $0.75 \mathrm{~mL} / \mathrm{min})$, retention time: $\mathrm{t}_{\mathrm{S}}=8.2 \mathrm{~min}$, and $\mathrm{t}_{\mathrm{R}}=9.2 \mathrm{~min} ;[\alpha]_{\mathrm{D}}^{23}-95.3\left(c 2.1, \mathrm{CHCl}_{3}\right)$; all other 
spectroscopic data as described above for 1-(2',2',2'-trichloromethylcarbonylamino)cyclohexa-2ene 6.

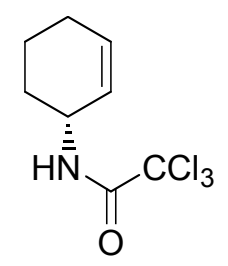

(1R)-(2',2',2'-Trichloromethylcarbonylamino)cyclohexa-2-ene 15. The reaction was carried out according general procedure 3 using (2E)-octa-2,7-dien-1-ol $3(0.08 \mathrm{~g}, 0.6 \mathrm{mmol})$ and $(R)$-COP-Cl $(0.04 \mathrm{~g}, 0.03 \mathrm{mmol})$ for the aza-Claisen rearrangement. Purification by flash column chromatography (elution with petroleum ether/ diethyl ether 97:3) gave (1R)-(2',2',2'trichloromethylcarbonylamino)cyclohexa-2-ene 15 as a white solid $(0.12 \mathrm{~g}, 75 \%$ yield over 3 steps). $88 \%$ ee determined by HPLC analysis using Chiracel-OD column $(0.5 \% \mathrm{iPrOH} /$ hexane at $0.75 \mathrm{~mL} / \mathrm{min})$, retention time: $\mathrm{t}_{\mathrm{S}}=8.3 \mathrm{~min}$, and $\mathrm{t}_{\mathrm{R}}=9.4 \mathrm{~min} ;[\alpha]_{\mathrm{D}}^{23}+97.4\left(c \mathrm{c} 1.3, \mathrm{CHCl}_{3}\right)$; all other spectroscopic data as described above for 1-(2',2',2'-trichloromethylcarbonylamino)cyclohexa-2ene 6.

\section{References:}

(1) Pinacho Crisóstomo, F. R.; Carillo, R.; León, L. G.; Martín, T.; Padrón, J. M.; Martín, V. S. J. Org. Chem. 2006, 71, 2339-2345.

(2) Ryu, J.-S.; Marks, T. J.; McDonald, F. E. J. Org. Chem. 2004, 69, 1038-1052.

(3) Ghosh, A. K.; Swanson, L. M.; Cho, H; Leshchenko, S.; Hussain, K. A.; Kay, S.; Walters, D. E.; Koh, Y.; Mitsuya, H. J. Med. Chem. 2005, 48, 3576-3585.

(4) Trehen, I. R.; Vig, R.; Singh, V.; Sharma, S.; Kad, G. L. Ind. J. Chem. 1992, 31B, 257-259.

(5) Castejón, P.; Moyano, A.; Pericàs, M. A.; Riera, A. Synth. Comm. 1994, 24, 1231-1238.

(6) Overman, L. E. J. Am. Chem. Soc. 1976, 98, 2901-2910.

(7) Donohoe, T. J.; Blades, K.; Moore, P. R.; Waring, M. J.; Winter, J. J. G.; Helliwell, M.; Newcombe, N. J.; Stemp, G. J. Org. Chem. 2002, 67, 7946-7956. 
$000^{\circ} 0-$

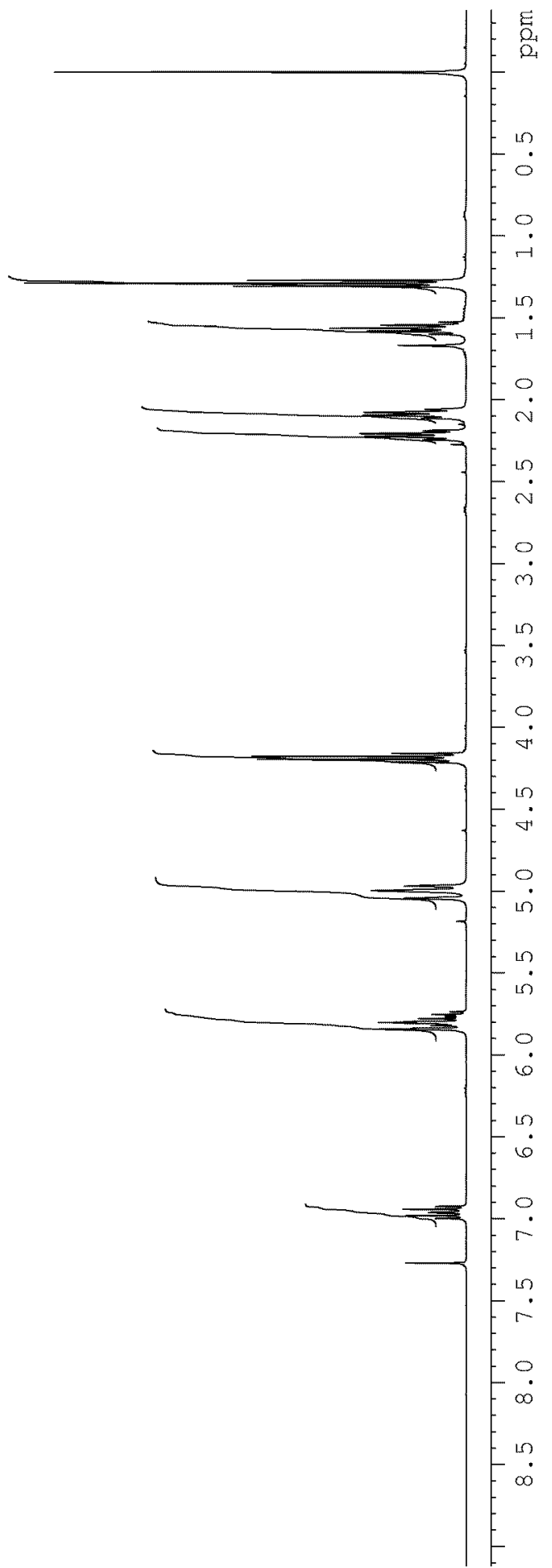


$900 \cdot 0-$

$082 \cdot 7$

$6 \varepsilon \mathrm{I} \cdot L Z$

$967 \cdot \tau \varepsilon=$

$6 \angle 0^{\circ} \varepsilon \varepsilon-$

$69 I \cdot 09$

OTL $9 L$ $\angle Z O^{\circ} L L$

五古 $\cdot L L$

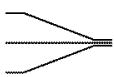

ZOT·GTI

gIG IZ

$220 \cdot 8 \varepsilon T$

$896 \cdot 87^{1}$

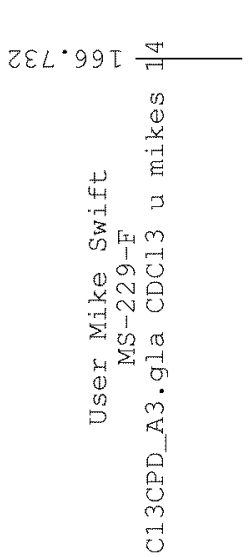

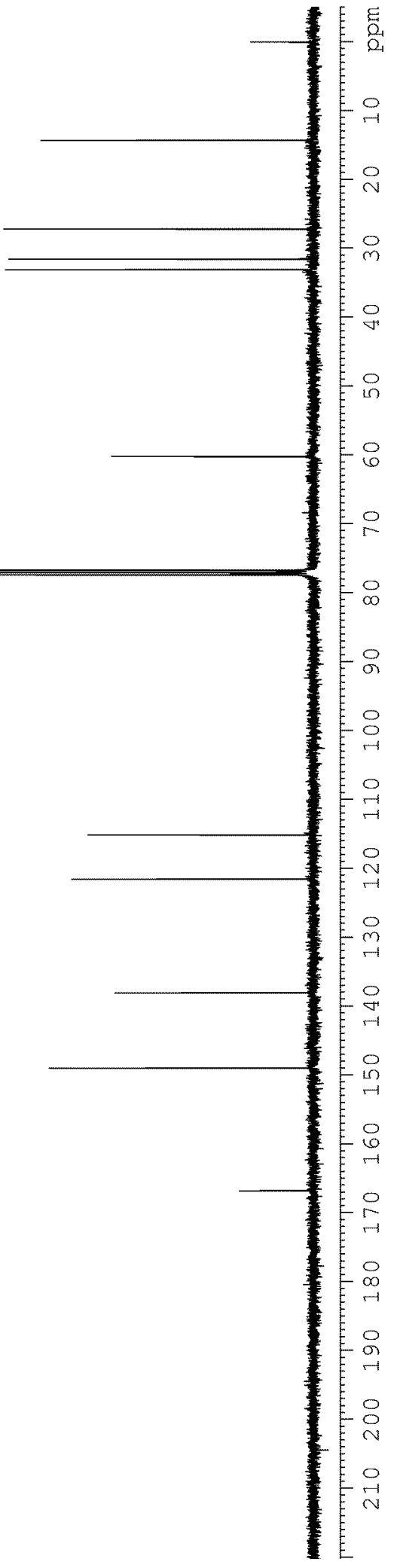



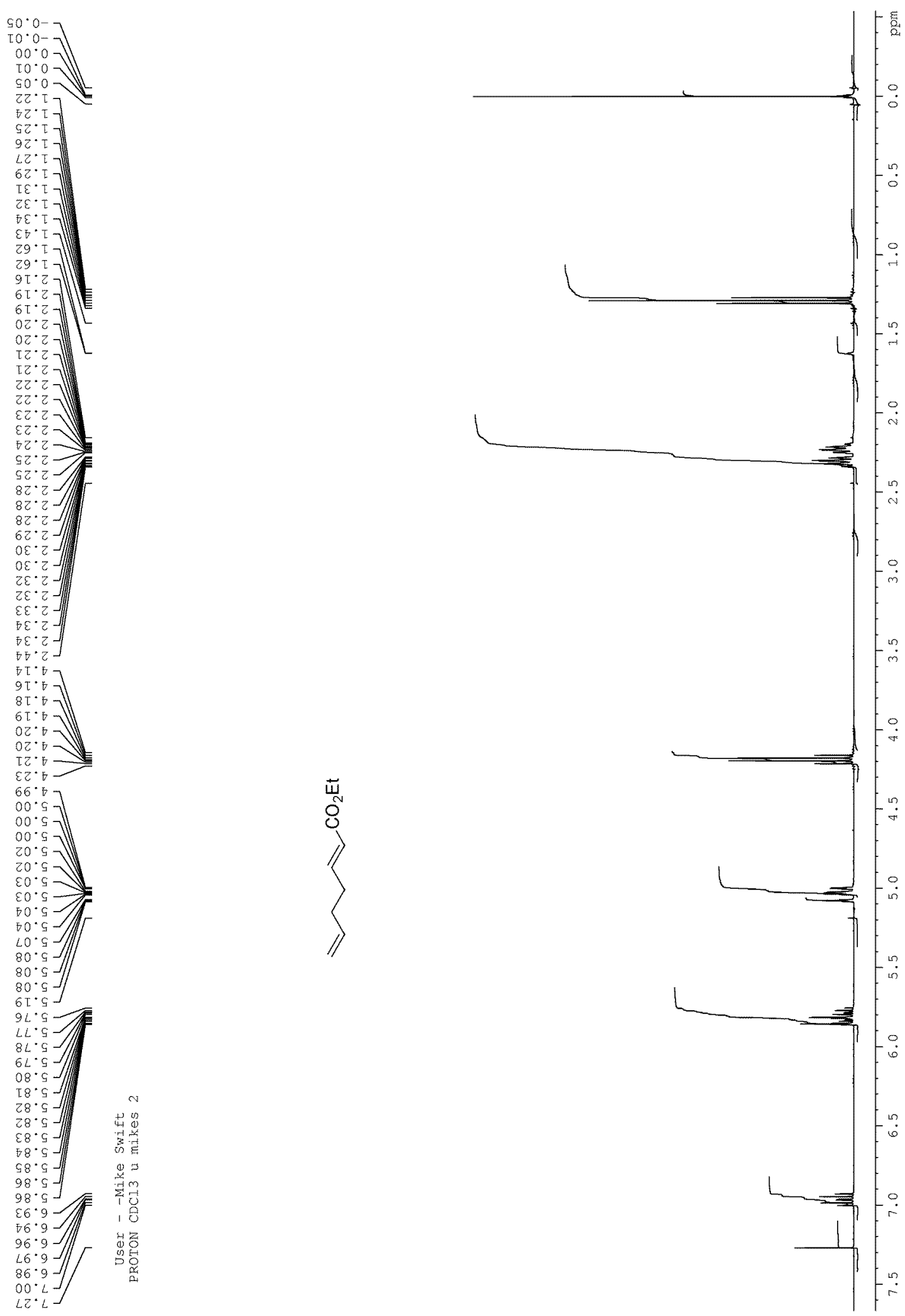


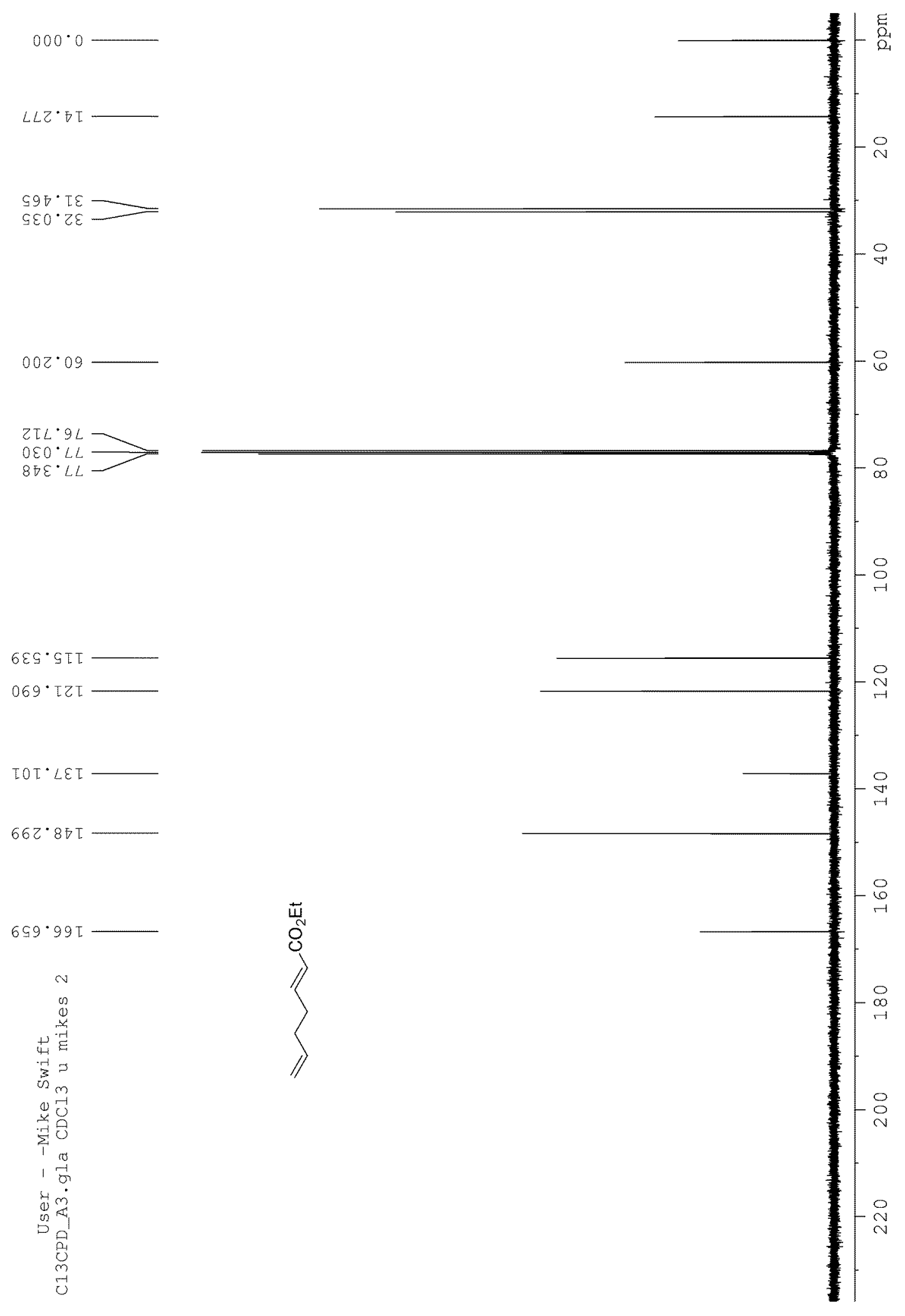



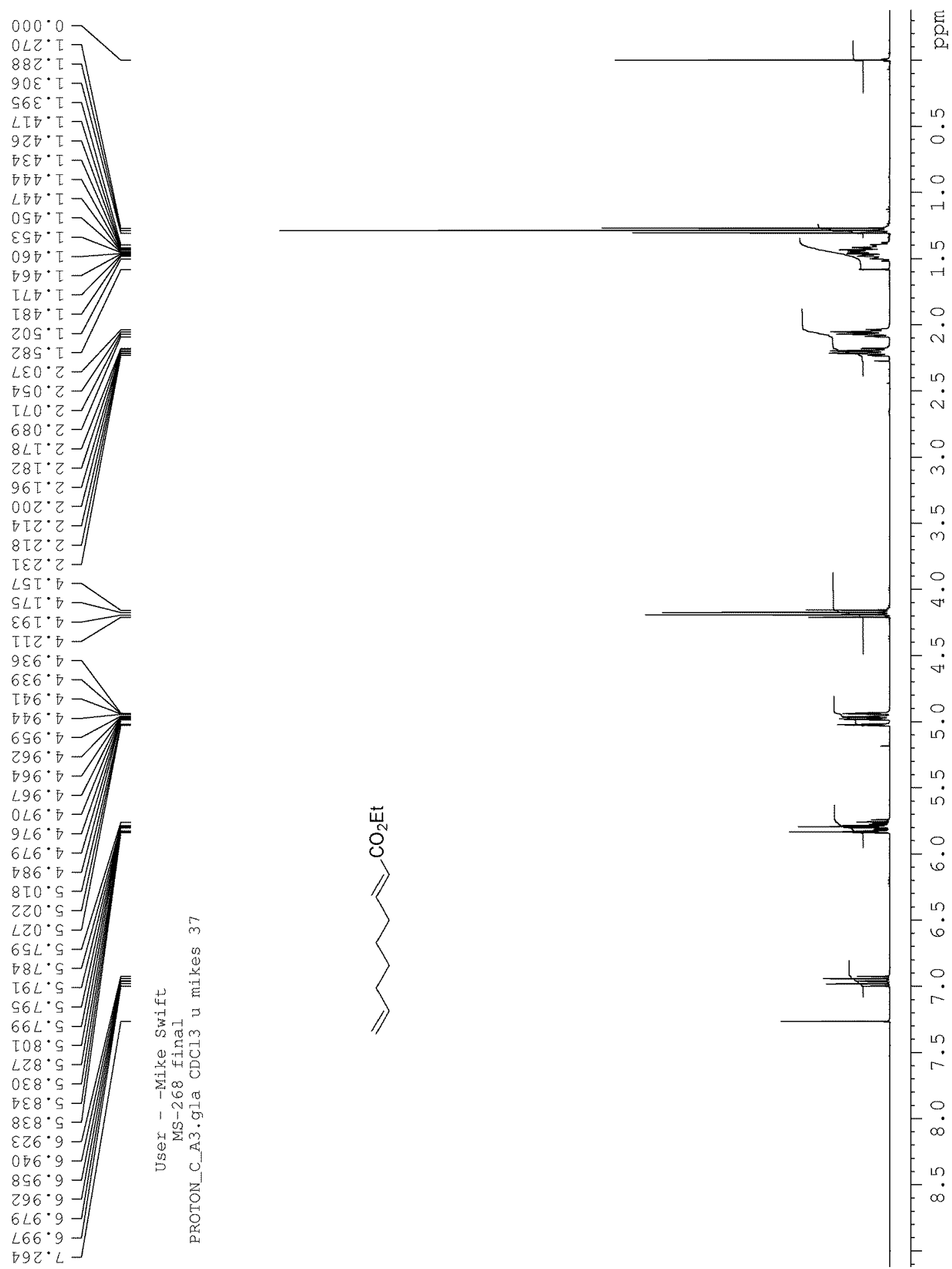

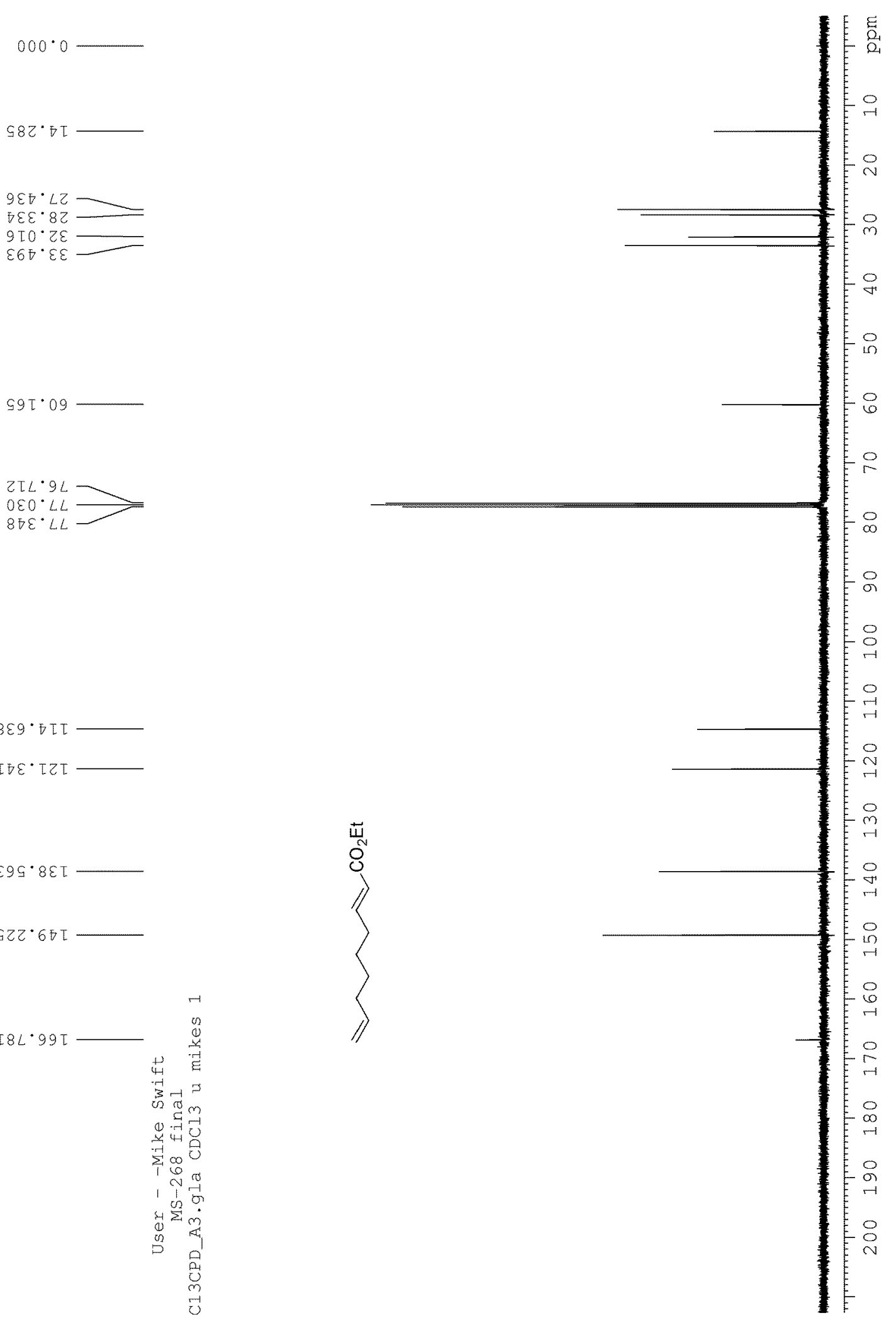


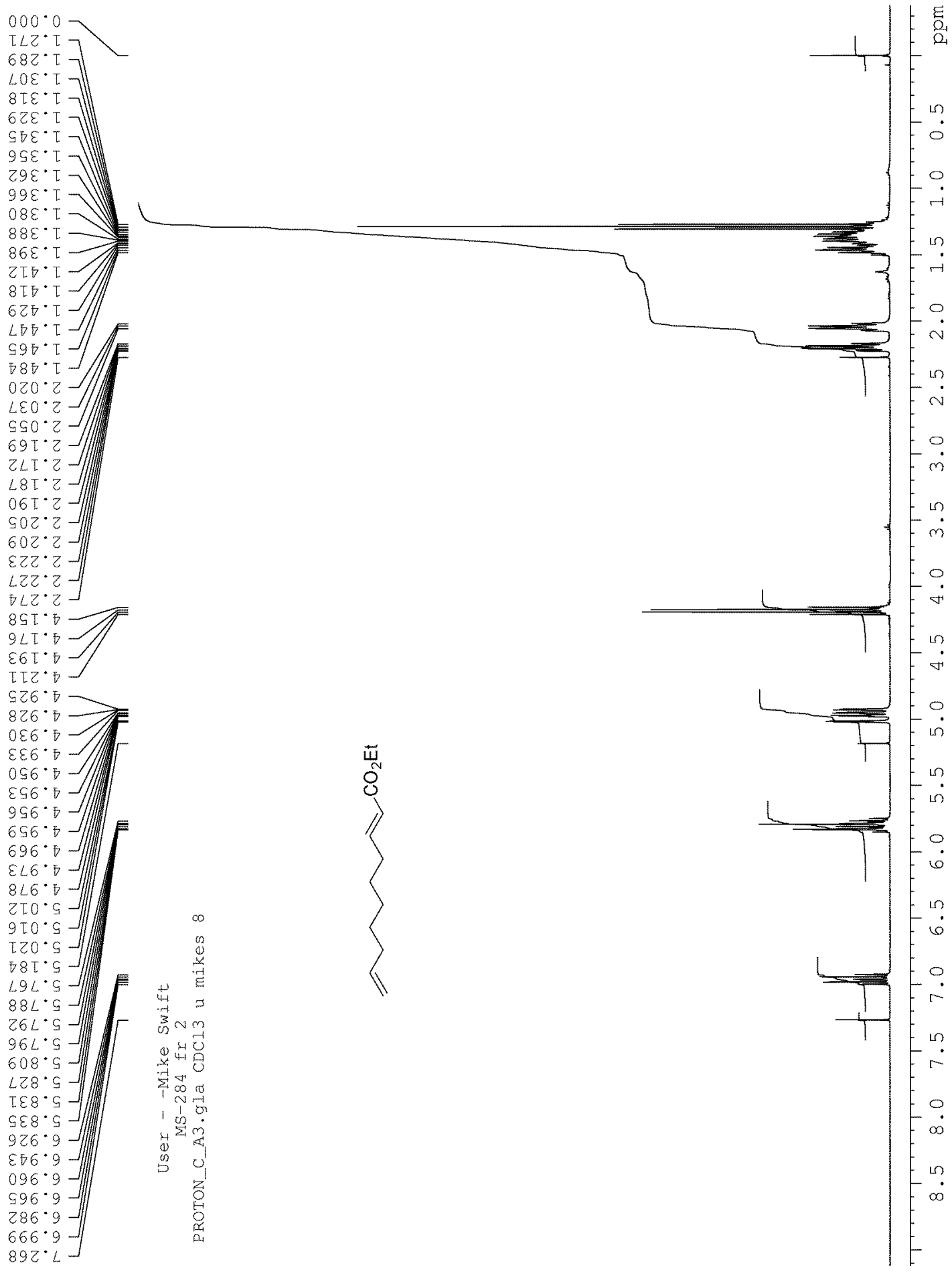




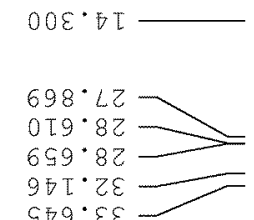

$90 \mathrm{I} \cdot 2 \varepsilon=$

$979^{\circ} \varepsilon \varepsilon$

T9I 09

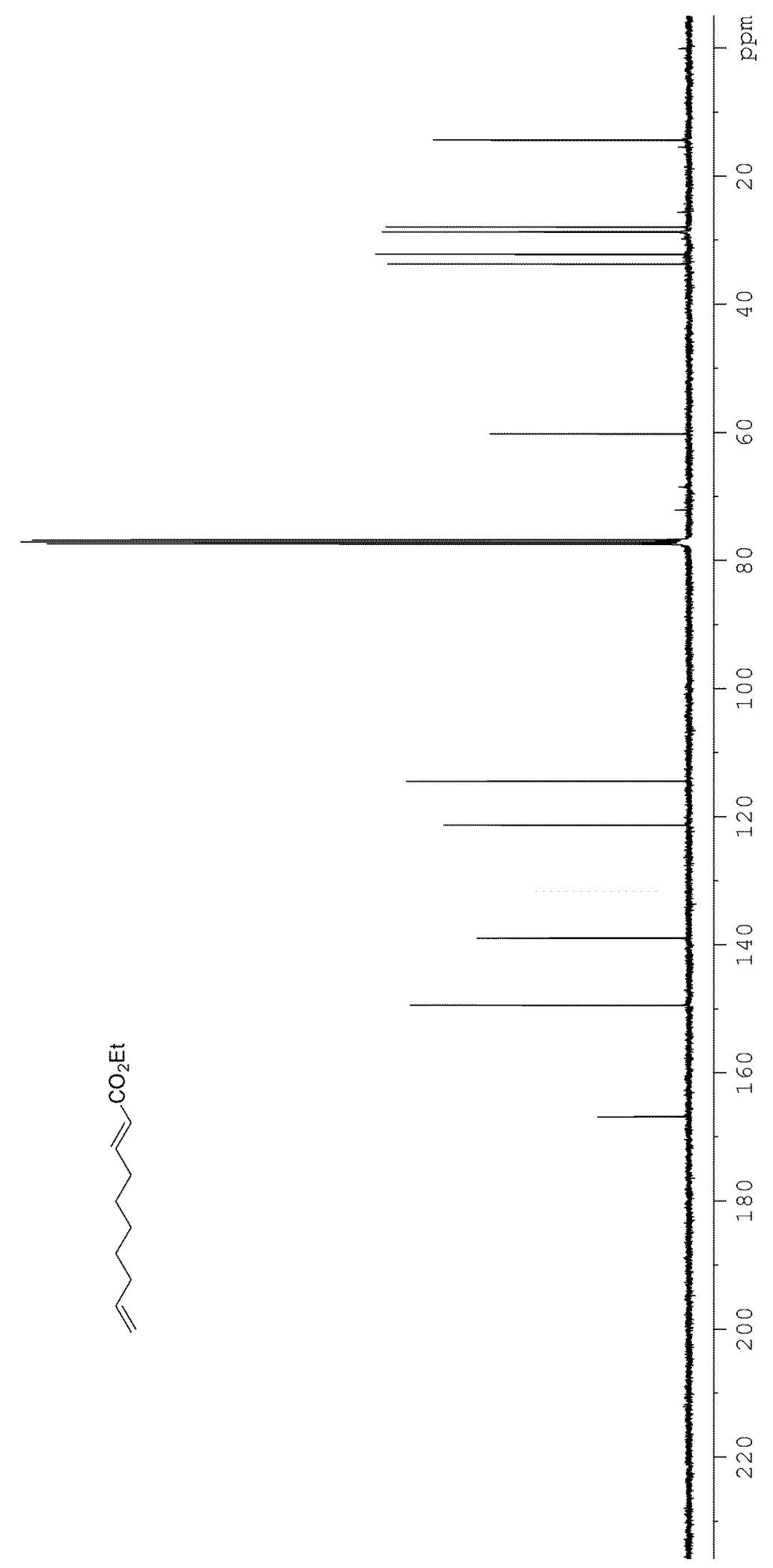

$82 L \cdot 9 L$

$900 \cdot L L \longrightarrow$

SOF'DTL

$28 Z^{*} \mathrm{IZT}$

$468 \cdot 8 \varepsilon T$

$9 \angle \varepsilon \cdot 6 \mathrm{~T} T$

$608 \cdot 99 I$

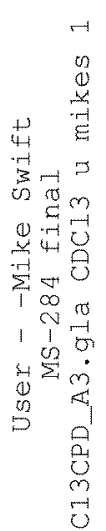




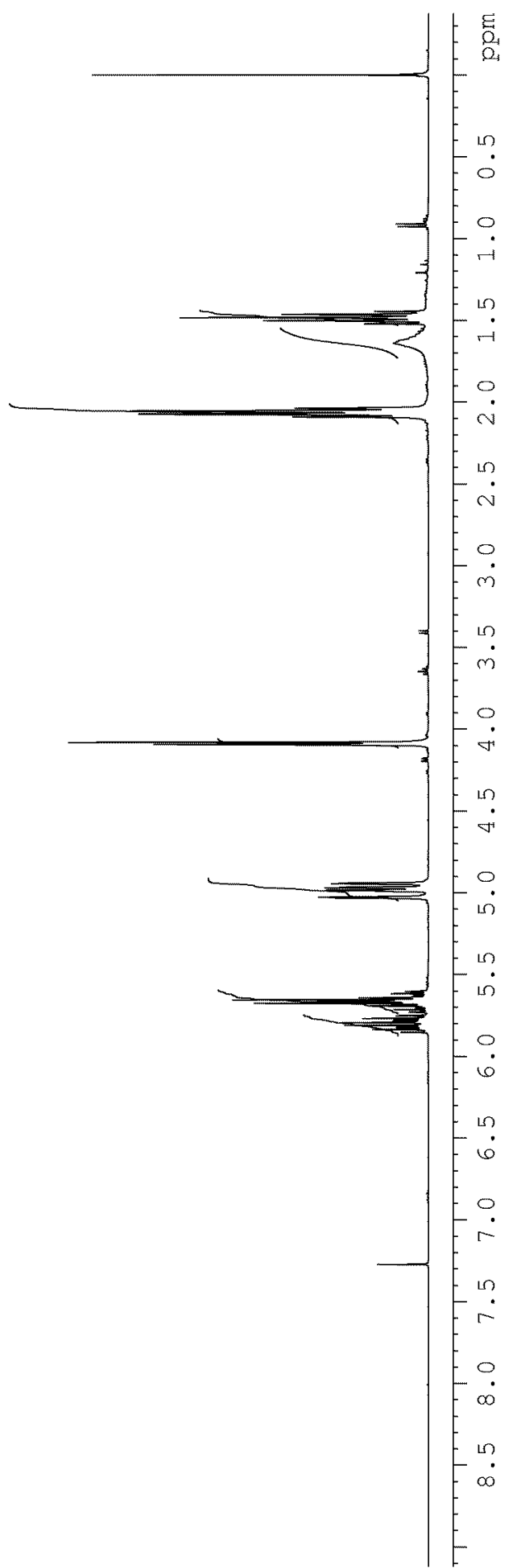


$500 \cdot 0-$
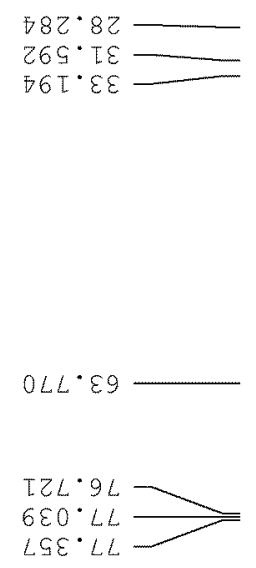

929・古垊

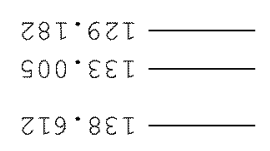

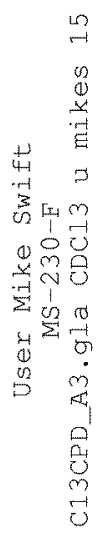

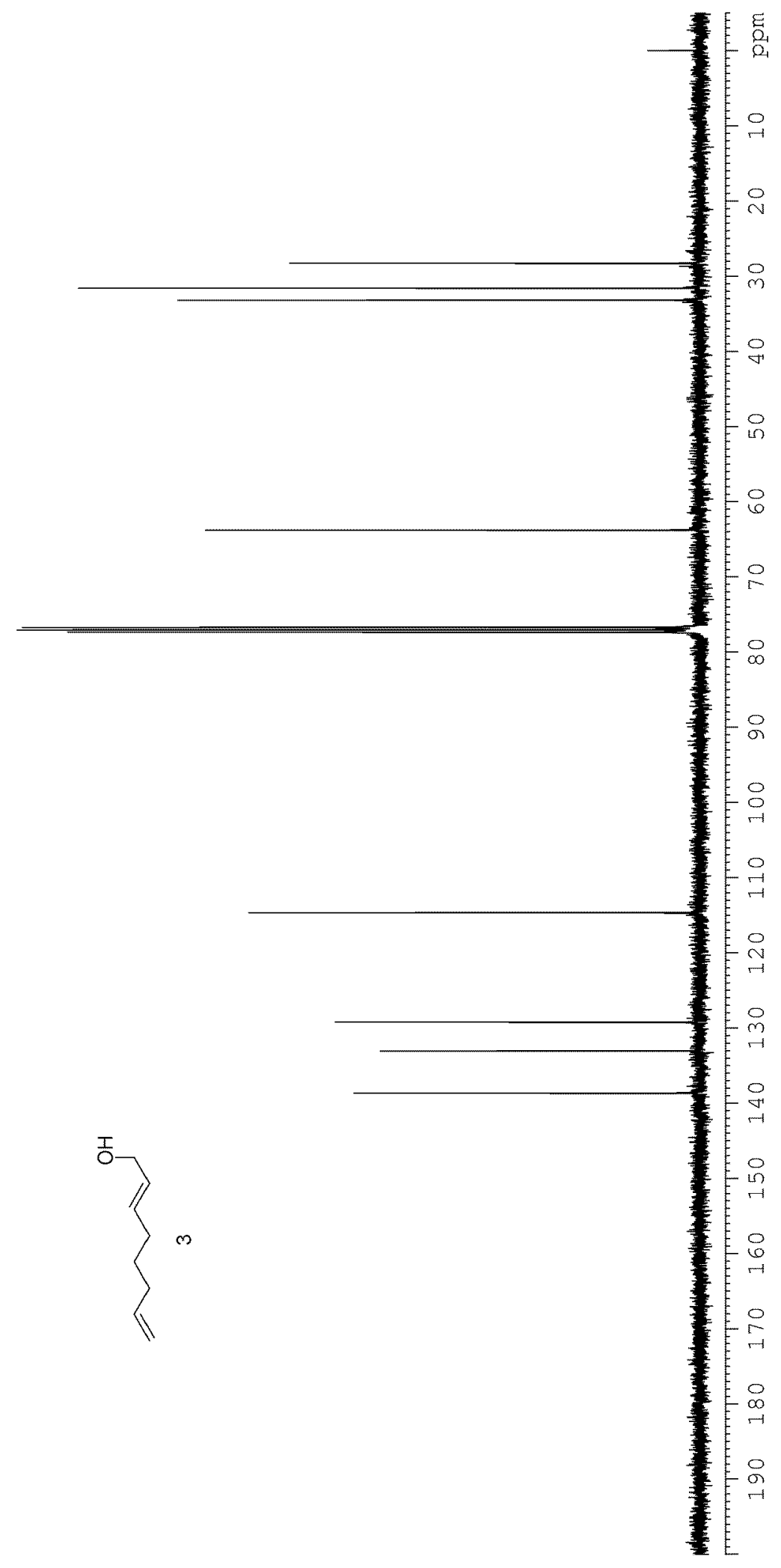

S19 


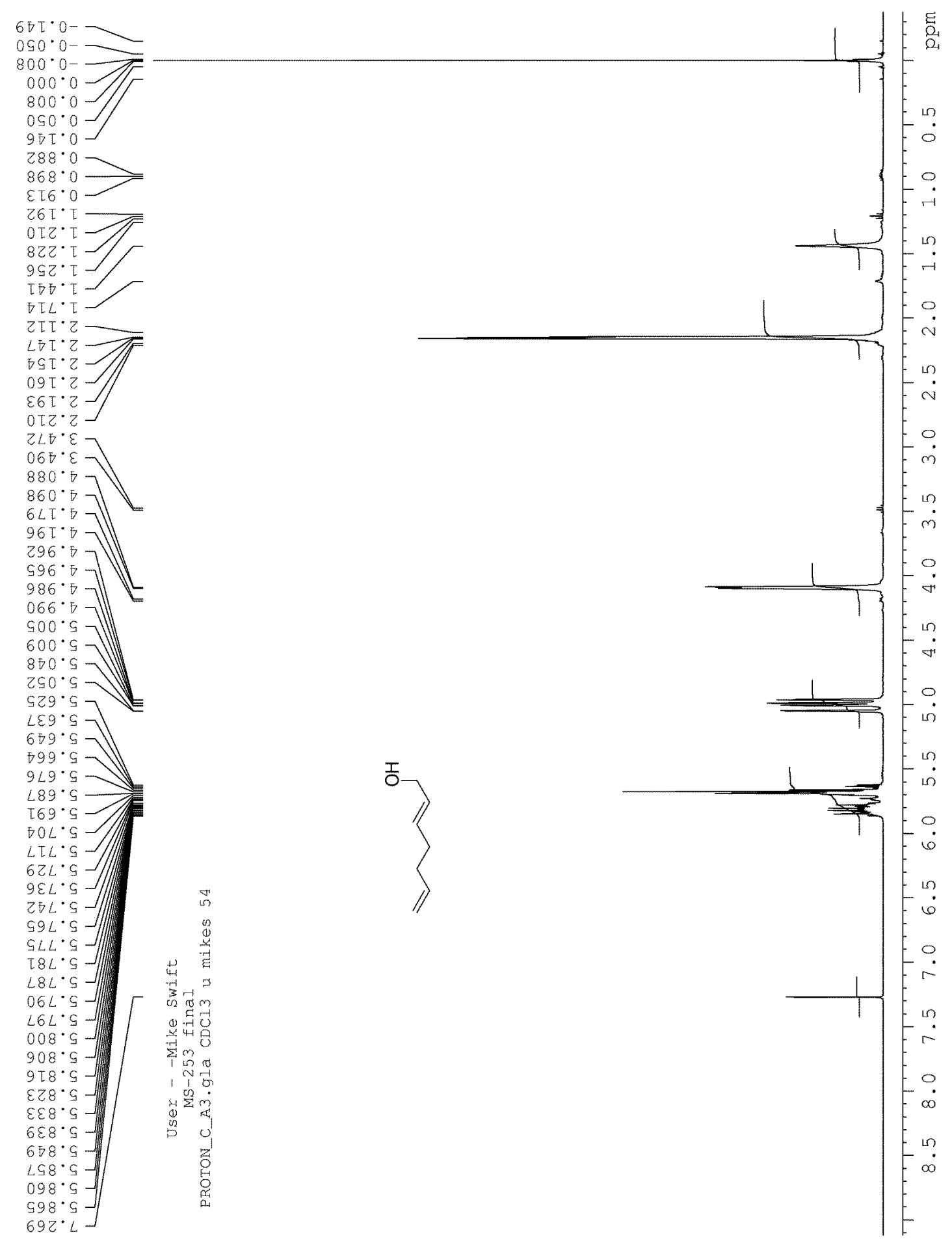




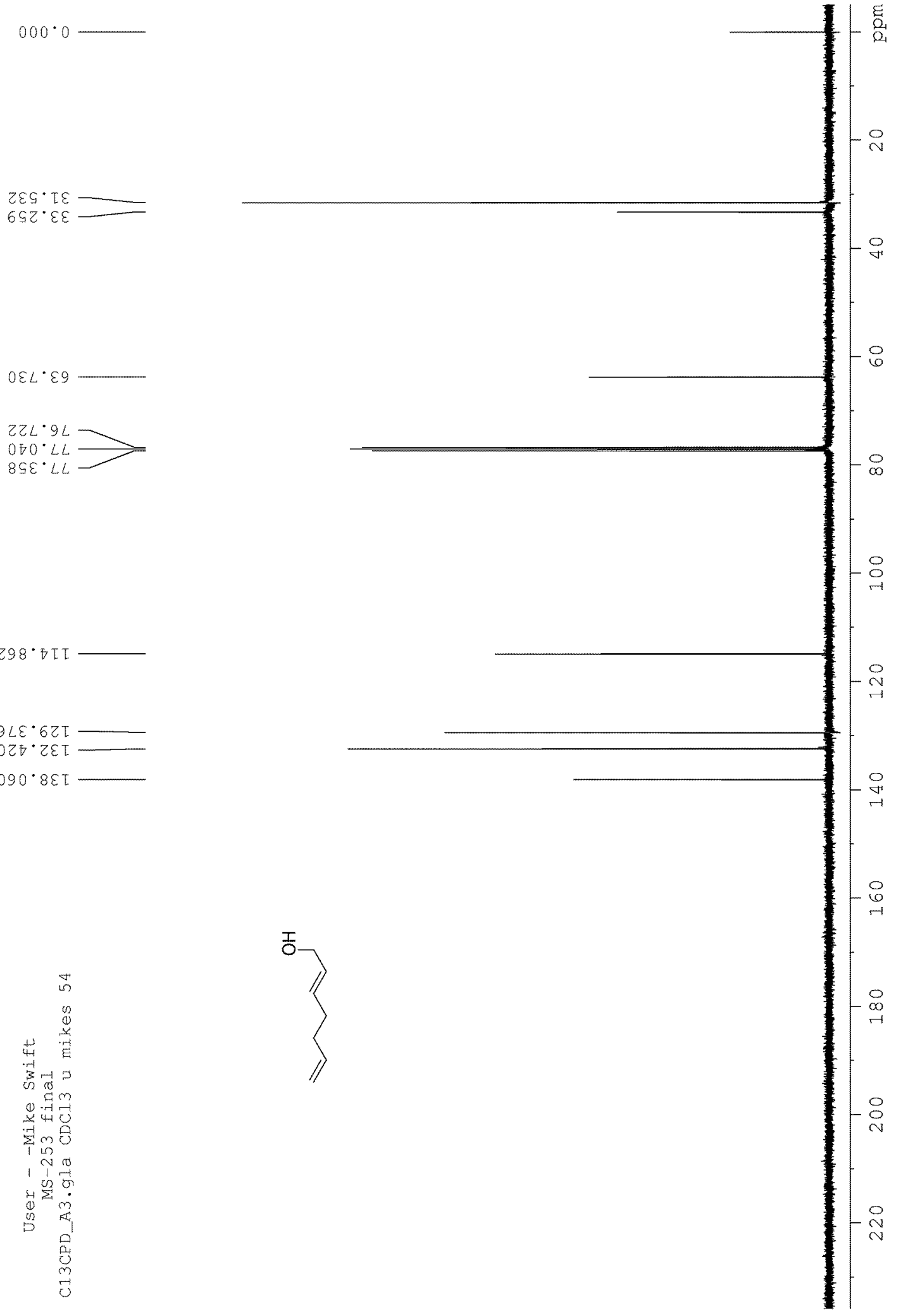




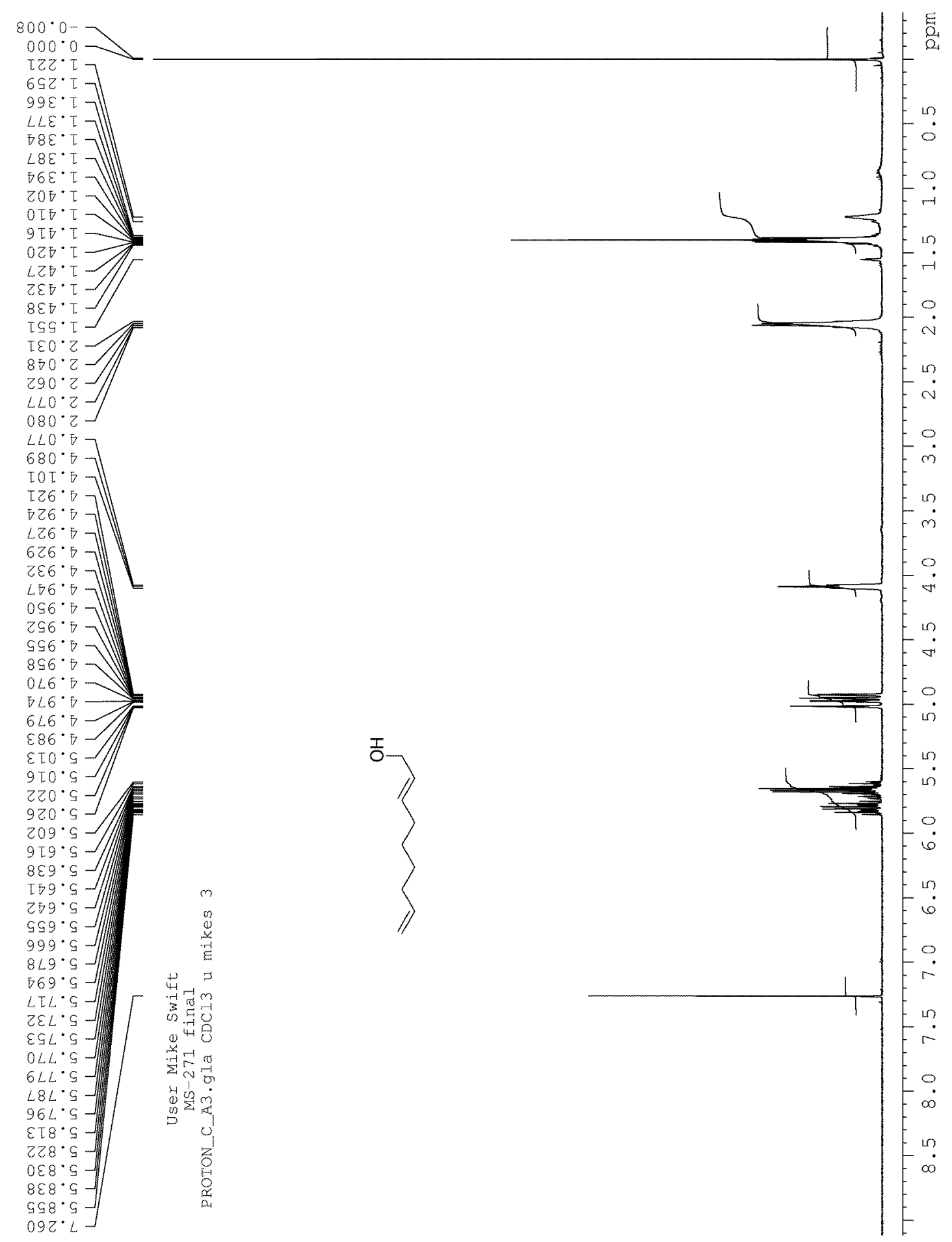




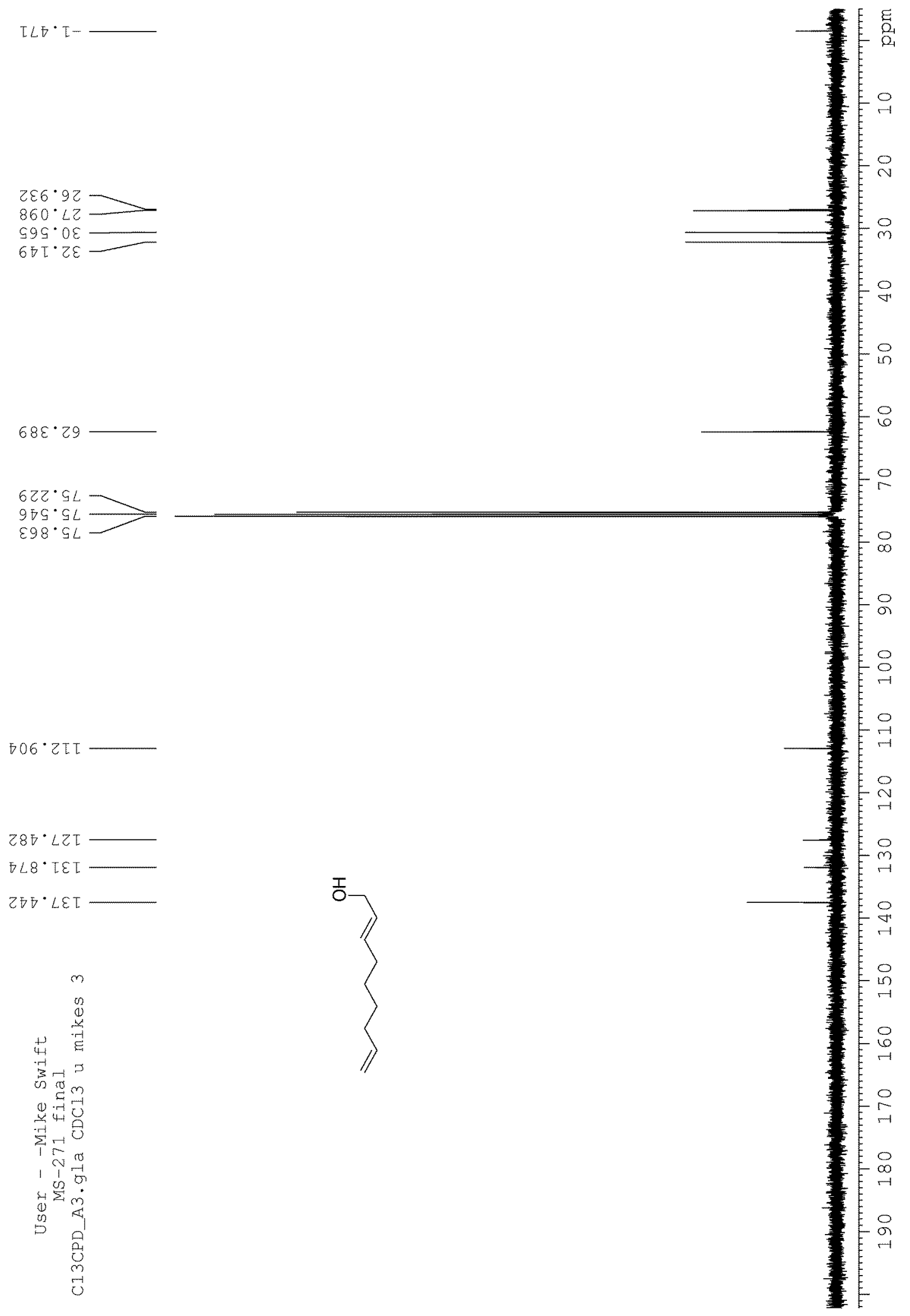



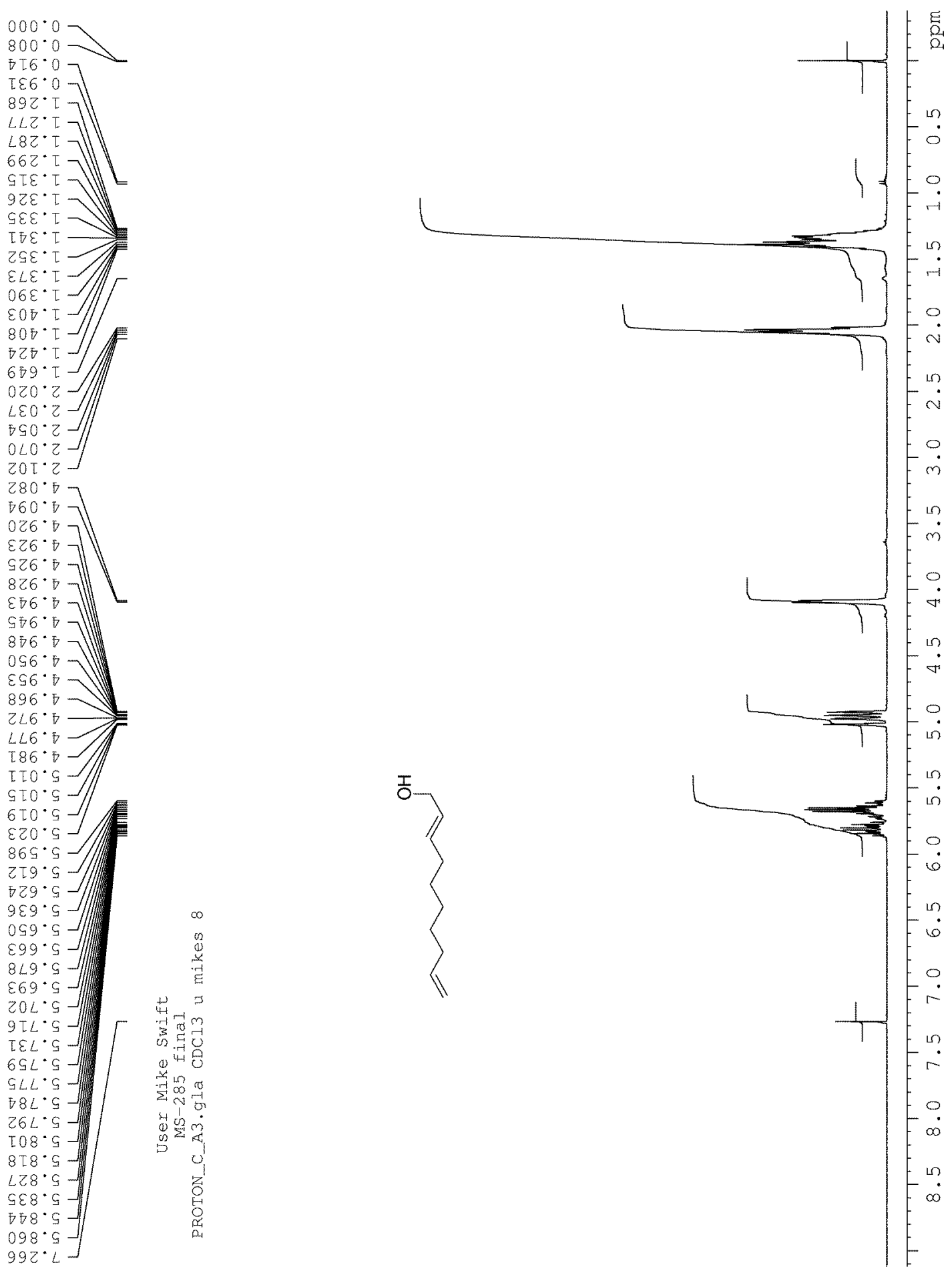


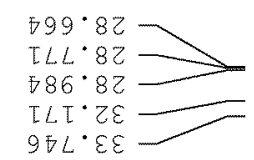

$8 L 8 \cdot \varepsilon 9$

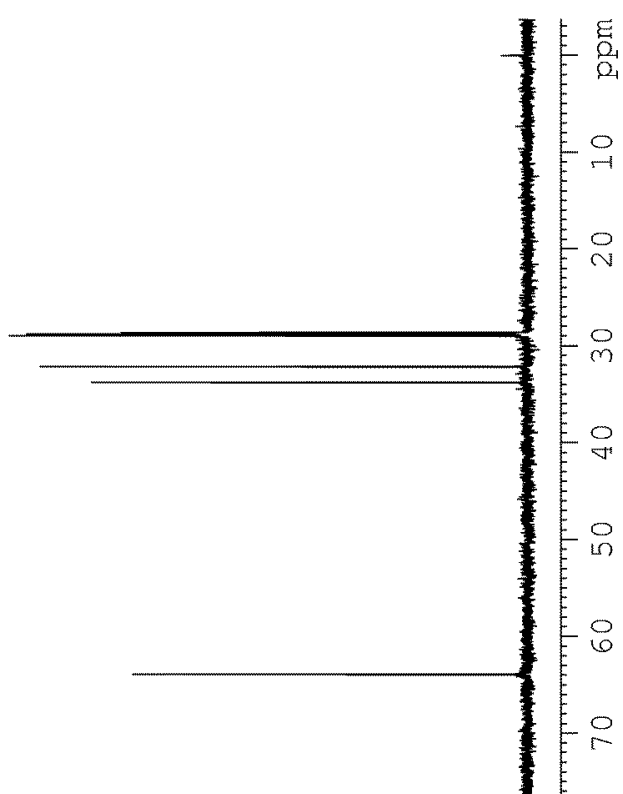

$9 \varepsilon L^{\circ} 9 L D$
$\varepsilon S O^{\circ} \angle L L$
$T \angle E \cdot L L$

$99 z \cdot 6 T T$

$268 \cdot 82 \mathrm{~T}$

$86)^{\circ} \varepsilon \varepsilon \mathrm{L}$

五项 $6 \varepsilon \tau$
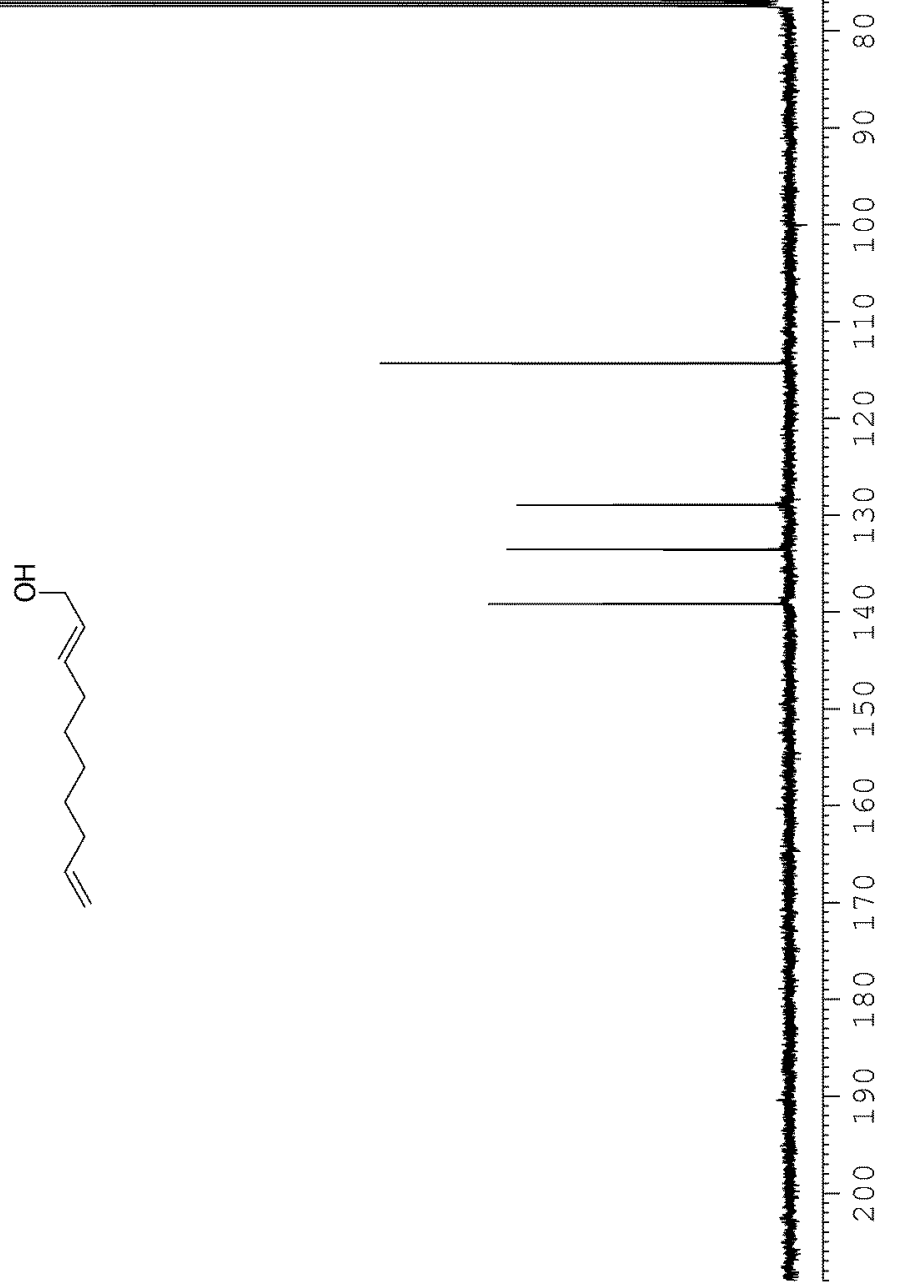

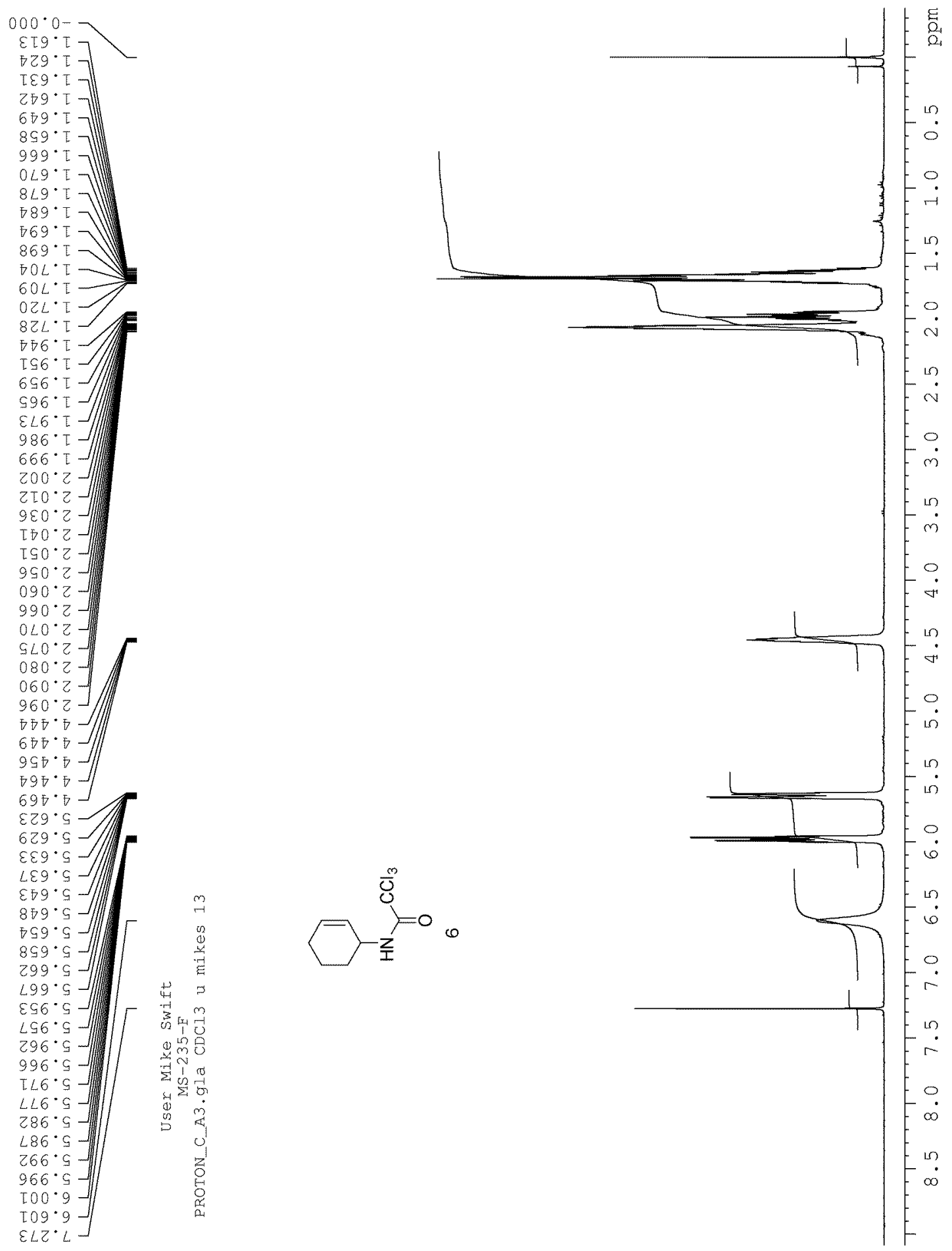

S26 
$607 \cdot 61$
$\angle 0 L \cdot 72$
$\angle 99 \cdot 82$

$968 \cdot 97$

TEL.9L
$670^{\circ} L L$
$99 \varepsilon^{\circ} L L$

$59 L \cdot 26$

$669^{\circ} 92 \mathrm{~T}$

$999^{\circ} 2 \varepsilon \mathrm{I}$

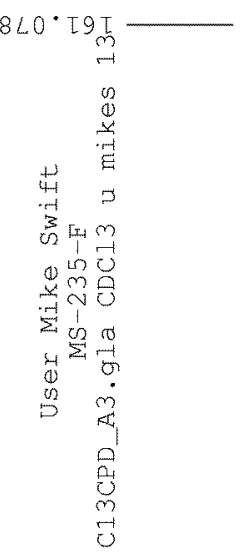

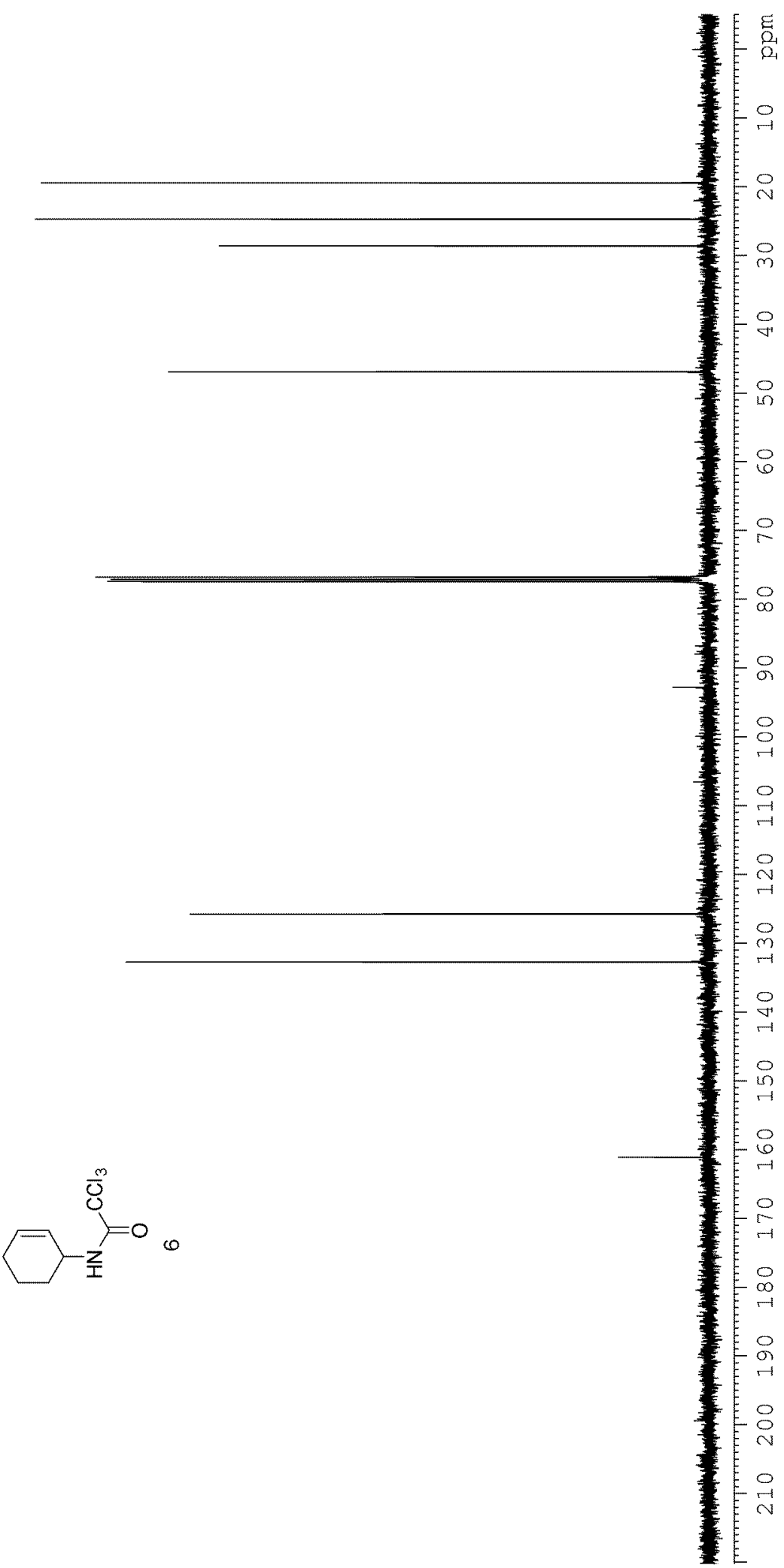




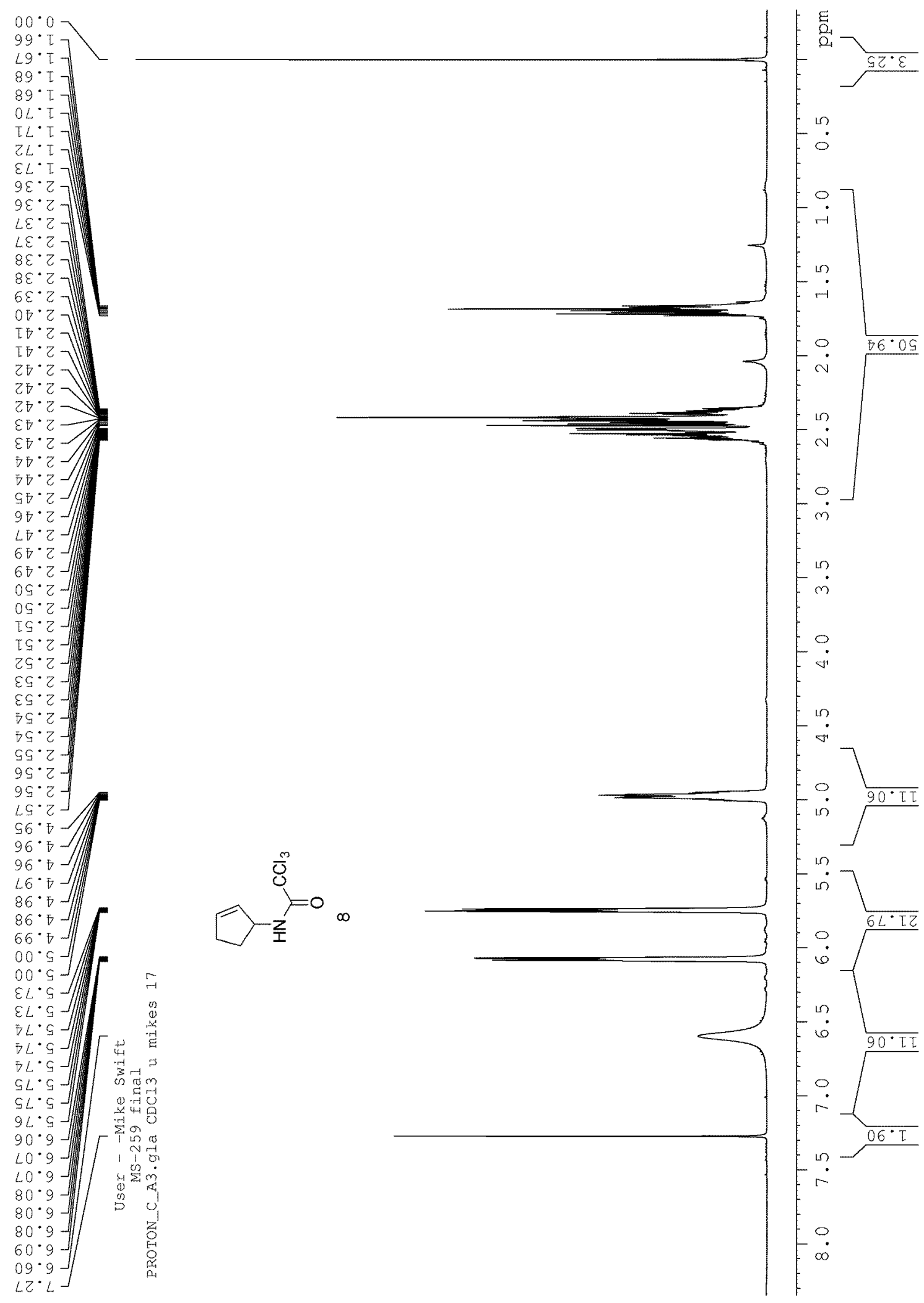


$968.82=$

ET0.99

$9[6 \cdot \bar{D} L$

$\nabla E Z \cdot S L$

$\operatorname{ESS}^{\circ} \operatorname{SL}$

T08 06

$G G \cdot \angle Z T$

$\mathrm{TO9}^{\circ} \mathrm{\vee} \varepsilon \mathrm{I}$

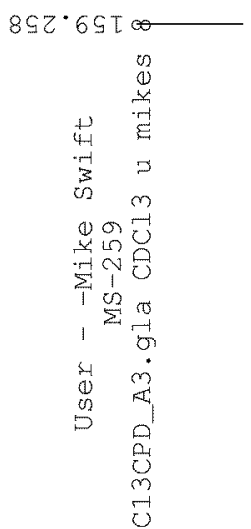

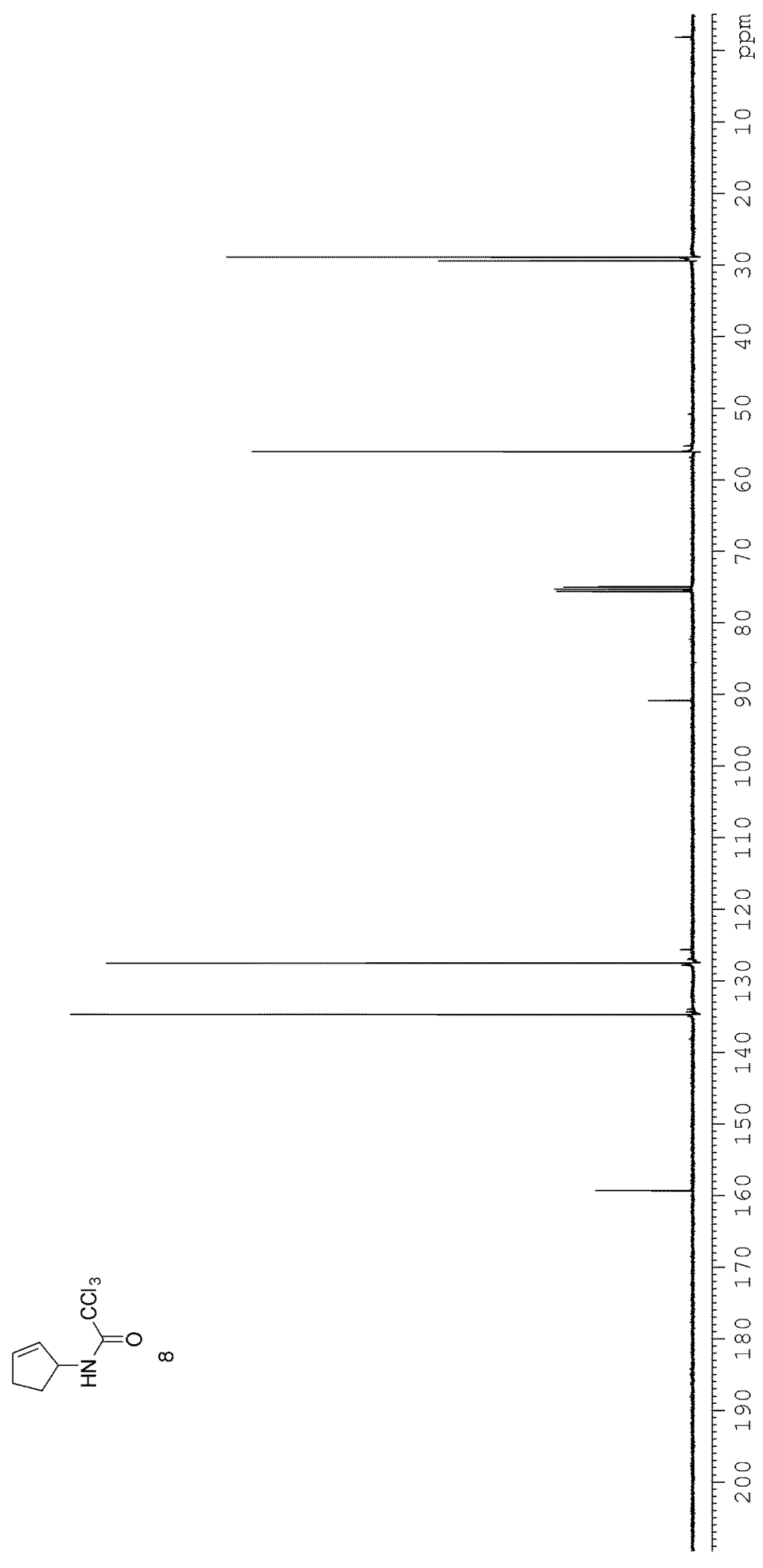




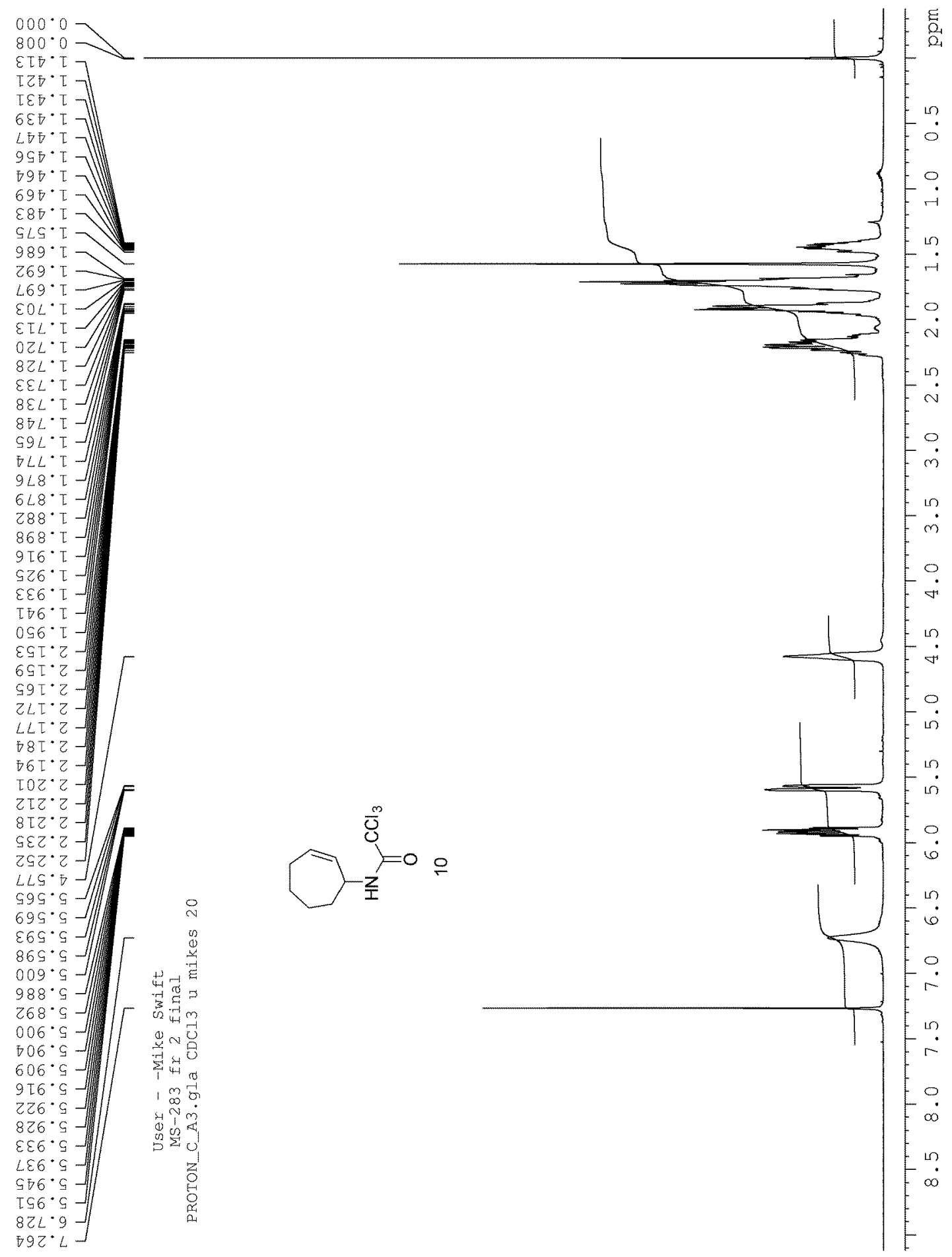




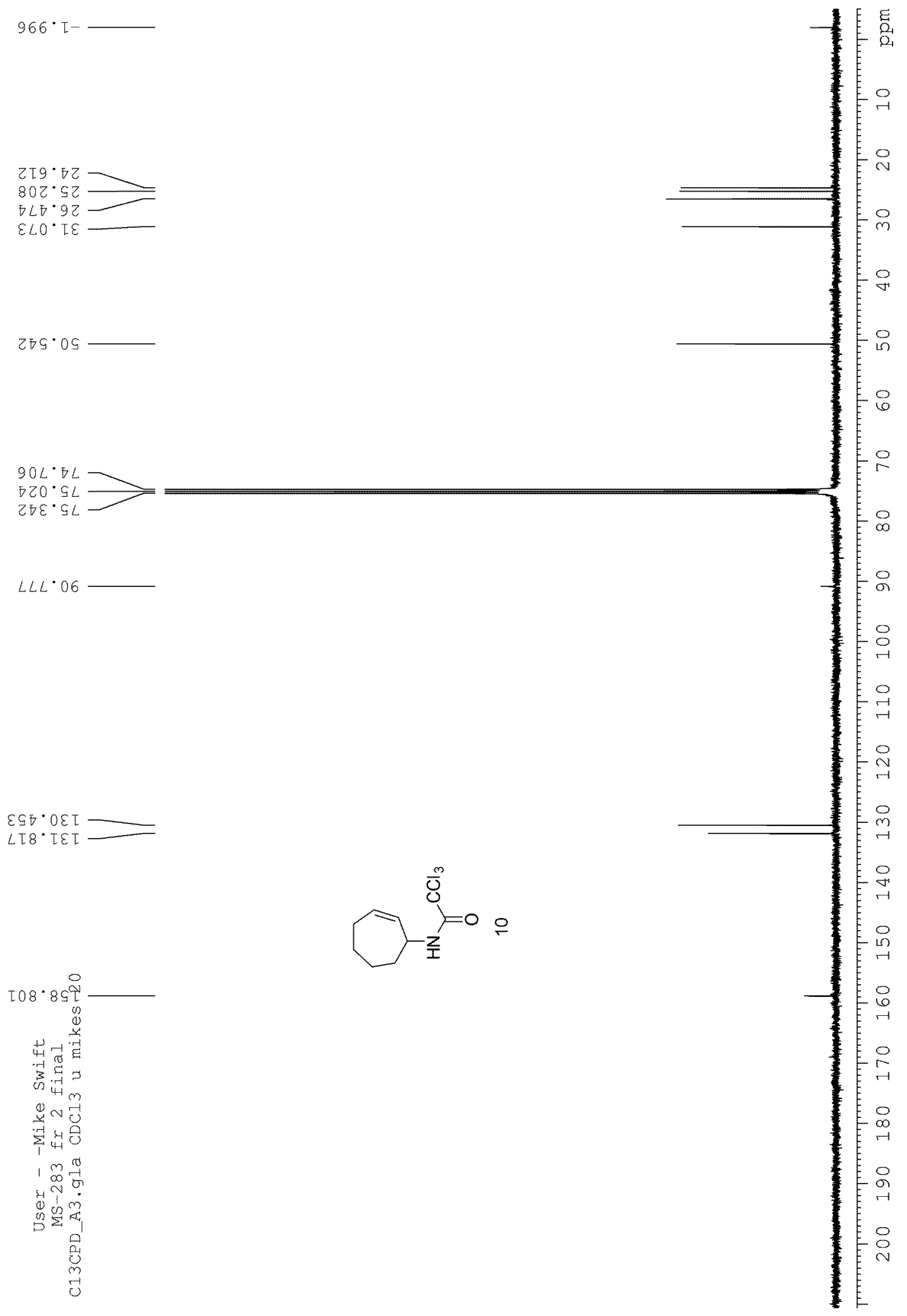




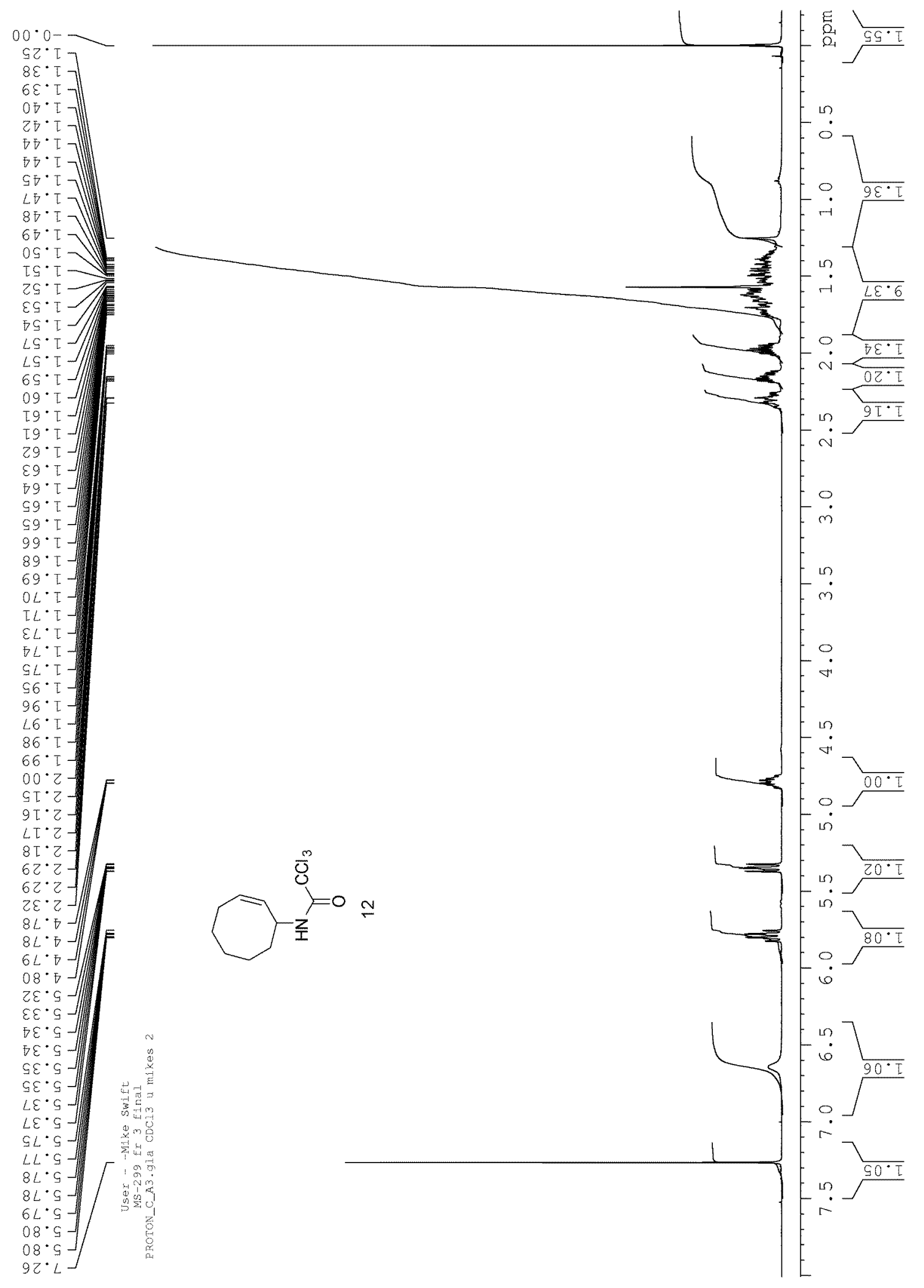


$0 I \cdot 2-$

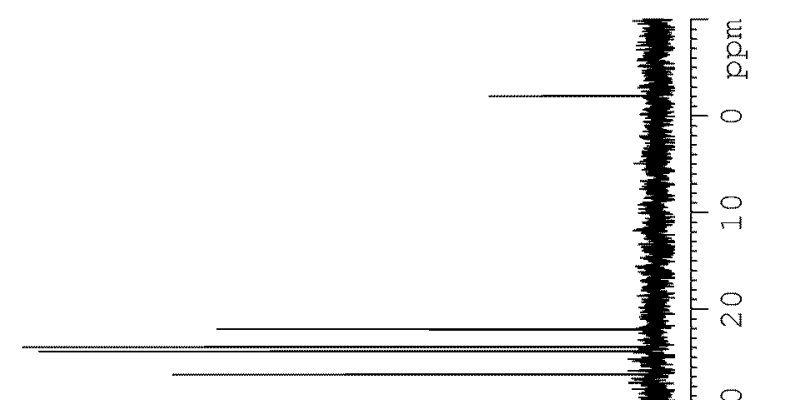

$L 0 \cdot 2 z=$
$68 \cdot \varepsilon z=$
$L \varepsilon \cdot 8 z=$
$9 L \cdot 9 z=$

$99 \cdot \varepsilon \varepsilon$

$88^{\circ} \angle 7$

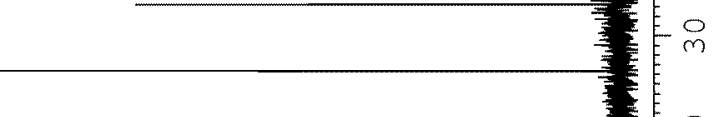

$26 \cdot 7 L \longrightarrow$

$99 \cdot 06 \square$
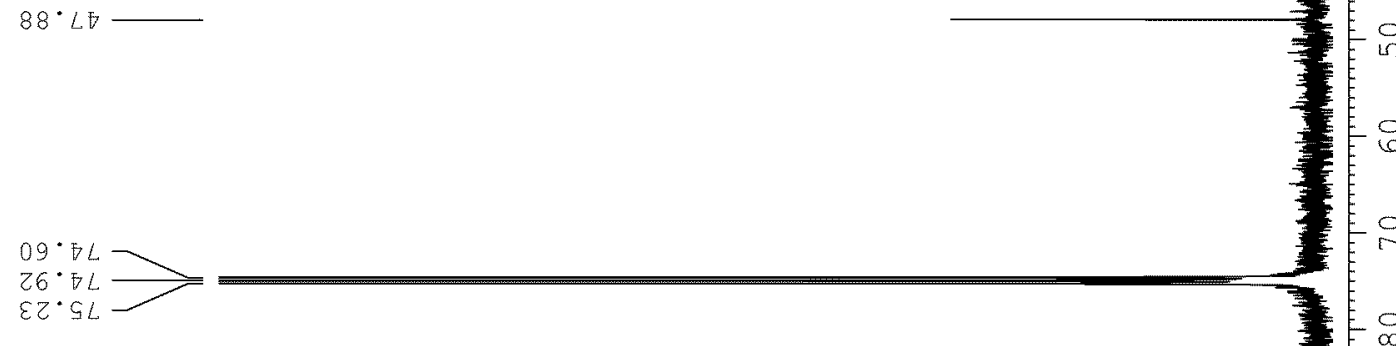

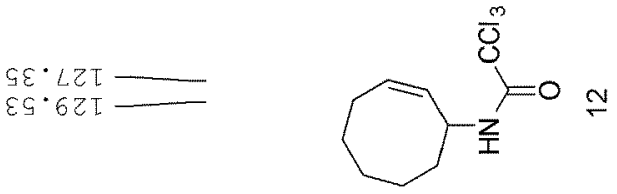

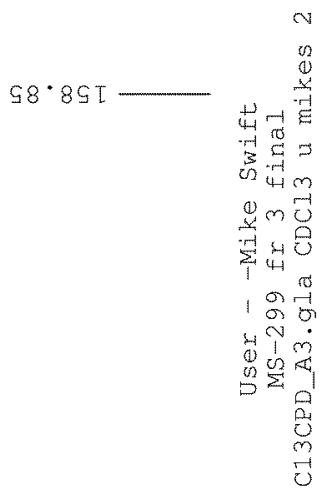

